ALEA, Lat. Am. J. Probab. Math. Stat. 15, 409-428 (2018)

DOI: 10.30757/ALEA.v15-17

\title{
Almost sure growth of supercritical multi-type continuous-state branching process
}

\author{
Andreas E. Kyprianou, Sandra Palau and Yan-Xia Ren \\ Department of Mathematical Sciences, University of Bath, \\ Claverton Down, Bath, BA2 7AY, UK. \\ E-mail address: a.kyprianou@bath.ac.uk \\ Department of Mathematical Sciences, University of Bath, \\ Claverton Down, Bath, BA2 7AY, UK. \\ E-mail address: sp2236@bath.ac.uk@bath.ac.uk \\ LMAM School of Mathematical Sciences \& Center for Statistical Science, \\ Peking University, Beijing, 100871, P.R. China \\ E-mail address: yxren@math.pku.edu.cn
}

\begin{abstract}
In Li (2011, Example 2.2), the notion of a multi-type continuous-state branching process (MCSBP) was introduced with a finite number of types, with the countably infinite case being proposed in Kyprianou and Palau (2018+). One may consider such processes as a super-Markov chain on a countable state-space of types, which undertakes both local and non-local branching. In Kyprianou and Palau (2018+) it was shown that, for MCSBPs, under mild conditions, there exists a lead eigenvalue which characterises the spectral radius of the linear semigroup associated to the process. Moreover, in a qualitative sense, the sign of this eigenvalue distinguishes between the cases where there is local extinction and exponential growth. In this paper, we continue in this vein and show that, when the number of types is finite, the lead eigenvalue gives the precise almost sure rate of growth of each type. This result matches perfectly classical analogues for multi-type GaltonWatson processes.
\end{abstract}

\section{Introduction}

Let $d \in \mathbb{N}$ be a natural number and put $E=\{1, \cdots, d\}$. For $\boldsymbol{x}=\left(x_{1}, \cdots, x_{d}\right)^{T} \in$ $\mathbb{R}^{d}$ we use the notation $x(i):=x_{i}$ for $i \in E$ and denote by $\|\boldsymbol{x}\|$ the Euclidean norm. We consider a multi-type continuous state branching process with $d$ types with branching mechanism $\psi$, henceforth referred to as a $\psi$-MCSBP. This is to say, we are interested in a $[0, \infty)^{d}$-valued strong Markov process $\boldsymbol{X}:=\left(\boldsymbol{X}_{t}, t \geq 0\right)$, with

Received by the editors September 29th, 2017; accepted February 15th, 2018.

2010 Mathematics Subject Classification. 60J68, 60J80.

Key words and phrases. Continuous-state branching processes, non-local branching mechanism, super Markov chain, strong law of large numbers. 
probabilities $\left\{\mathbb{P}_{\boldsymbol{x}}, \boldsymbol{x} \in \mathbb{R}_{+}^{d}\right\}$ such that the following branching property hold: for all $\boldsymbol{x}, \boldsymbol{y} \in \mathbb{R}_{+}^{d}$,

$$
\mathbb{E}_{\boldsymbol{x}+\boldsymbol{y}}\left[\mathrm{e}^{-\left\langle\boldsymbol{f}, \boldsymbol{X}_{t}\right\rangle}\right]=\mathbb{E}_{\boldsymbol{x}}\left[\mathrm{e}^{-\left\langle\boldsymbol{f}, \boldsymbol{X}_{t}\right\rangle}\right] \mathbb{E}_{\boldsymbol{y}}\left[\mathrm{e}^{-\left\langle\boldsymbol{f}, \boldsymbol{X}_{t}\right\rangle}\right], \quad t \geq 0, \quad \boldsymbol{f} \in \mathbb{R}_{+}^{d} .
$$

Its branching mechanism is a vectorial function $\psi: E \times \mathbb{R}_{+}^{d} \rightarrow \mathbb{R}^{d}$ such that

$\psi_{i}(\boldsymbol{u}):=-\left\langle\boldsymbol{u}, \widetilde{\boldsymbol{B}} \boldsymbol{e}_{i}\right\rangle+c_{i} u_{i}^{2}+\int_{\mathbb{R}_{+}^{d}}\left(\mathrm{e}^{-\langle\boldsymbol{u}, \boldsymbol{z}\rangle}-1+u_{i}\left(z_{i} \wedge 1\right)\right) \mu_{i}(\mathrm{~d} \boldsymbol{z}), \quad \boldsymbol{u} \in \mathbb{R}_{+}^{d}, i \in E$,

where $c_{i} \in \mathbb{R}_{+}, \widetilde{\boldsymbol{B}}$ is a $d \times d$ matrix such that $\widetilde{B}_{i, j} \mathbf{1}_{\{i \neq j\}} \in \mathbb{R}_{+}, \boldsymbol{e}_{\mathbf{1}}, \cdots, \boldsymbol{e}_{\boldsymbol{d}}$ is the natural basis in $\mathbb{R}^{d}$, and $\mu_{i}$ is a measure concentrated on $\mathbb{R}_{+}^{d} \backslash\{\mathbf{0}\}$ such that

$$
\int_{\mathbb{R}_{+}^{d}}\left[\|\boldsymbol{z}\| \wedge\|\boldsymbol{z}\|^{2}+\sum_{j \in E} \mathbf{1}_{\{j \neq i\}} z_{j}\right] \mu_{i}(\mathrm{~d} \boldsymbol{z})<\infty .
$$

The process $\boldsymbol{X}$ is characterized by its Laplace transform:

$$
\mathbb{E}_{\boldsymbol{x}}\left[\mathrm{e}^{-\left\langle\boldsymbol{f}, \boldsymbol{X}_{t}\right\rangle}\right]=\mathrm{e}^{-\langle\boldsymbol{x}, \boldsymbol{v}(t, \boldsymbol{f})\rangle}, \quad \boldsymbol{x}, \boldsymbol{f} \in \mathbb{R}_{+}^{d}, t \in \mathbb{R}_{+},
$$

where, for any $f \in \mathbb{R}_{+}^{d}$, the continuous differentiable function

$$
t \mapsto \boldsymbol{v}(t, \boldsymbol{f})=\left(v_{1}(t, \boldsymbol{f}), \cdots, v_{d}(t, \boldsymbol{f})\right)^{T},
$$

is the unique locally bounded non-negative solution to the system of integral equations

$$
v_{i}(t, \boldsymbol{f})=f_{i}-\int_{0}^{t} \psi_{i}(\boldsymbol{v}(s, \boldsymbol{f})) \mathrm{d} s, \quad i \in E .
$$

According to Barczy et al. (2015), this process can be seen as a strong solution of a stochastic differential equation (SDE). More precisely, let $\boldsymbol{W}_{t}$ be a $d$-dimensional standard Brownian motion, and for each $i \in E$, let $N_{i}$ be a Poisson random measure on $\mathbb{R}_{+} \times \mathbb{R}_{+}^{d} \times \mathbb{R}_{+}$with intensity measure $\mathrm{d} s \mu_{i}(\mathrm{~d} \boldsymbol{z}) \mathrm{d} r$, and denote by $\widetilde{N}_{i}$ its compensated measure. Suppose that $\boldsymbol{W}$ and $\left(N_{i}\right)_{i \in E}$ are independent of each other. Then, a MCSBP with branching mechanism $\psi$ is characterized as the unique $\mathbb{R}_{+}^{d}$-valued strong solution to the SDE

$$
\begin{aligned}
\boldsymbol{X}_{t}= & \boldsymbol{X}_{0}+\int_{0}^{t} \boldsymbol{B} \boldsymbol{X}_{s} \mathrm{~d} s+\sum_{i \in E} \boldsymbol{e}_{i} \int_{0}^{t} \sqrt{2 c_{i} X_{s, i}} \mathrm{~d} W_{s, i} \\
& +\sum_{i \in E} \int_{0}^{t} \int_{\mathbb{R}_{+}^{d}} \int_{0}^{\infty} \boldsymbol{z} \mathbf{1}_{\left\{r \leq X_{s-, i}\right\}} \widetilde{N}_{i}(\mathrm{~d} s, \mathrm{~d} \boldsymbol{z}, \mathrm{d} r),
\end{aligned}
$$

where the matrix $\boldsymbol{B}$ is given by

$$
B_{i, j}=\widetilde{B}_{i, j}+\int_{\mathbb{R}_{+}^{d}}\left(z_{i}-\delta_{i, j}\right)^{+} \mu_{j}(\mathrm{~d} \boldsymbol{z})
$$

Moreover, they proved in Barczy et al. (2015, Formula (2.15) and the later computations) that $\psi$ can be written as

$$
\psi_{i}(\boldsymbol{u})=-\left\langle\boldsymbol{u}, \boldsymbol{B} \boldsymbol{e}_{i}\right\rangle+c_{i} u_{i}^{2}+\int_{\mathbb{R}^{d}}\left(\mathrm{e}^{-\langle\boldsymbol{u}, \boldsymbol{z}\rangle}-1+\langle\boldsymbol{u}, \boldsymbol{z}\rangle\right) \mu_{i}(\mathrm{~d} \boldsymbol{z}), \quad \boldsymbol{u} \in \mathbb{R}_{+}^{d}, i \in E .
$$

We additionally assume that $\mathbb{P}_{\boldsymbol{e}_{\boldsymbol{i}}}\left(\left\langle 1, X_{t}\right\rangle=0\right)>0$ for all $t>0, i=1, \cdots, d$. 
Remark 1.1. Thanks to the relation between $\boldsymbol{B}$ and $\widetilde{\boldsymbol{B}}$, when we write the branching mechanism as in (1.4), the matrix $\boldsymbol{B}$ satisfies

$$
\int_{\mathbb{R}_{+}^{d}} z(i) \mu_{j}(\mathrm{~d} \boldsymbol{z}) \leq B_{i j} \quad \text { for all } i \neq j .
$$

Remark 1.2. If we regard $E$ as the space where particles located, the model we described above can be seen as a special case of a superprocess in which the associated Markov movement is that of a Markov chain on $E$. Indeed, for such a process, from e.g. Dynkin (1991), the log-Laplace semigroup, $\boldsymbol{V}_{t}: \mathbb{R}_{+}^{d} \mapsto \mathbb{R}_{+}^{d}$, which similarly to (1.1), satisfies $\left\langle\boldsymbol{x}, \boldsymbol{V}_{t} \boldsymbol{f}\right\rangle=-\log \mathbb{E}_{\boldsymbol{x}}\left[\mathrm{e}^{-\left\langle\boldsymbol{f}, \boldsymbol{X}_{t}\right\rangle}\right]$, for $\boldsymbol{f}, \boldsymbol{x} \in \mathbb{R}_{+}^{d}$, and is the unique solution to

$$
\boldsymbol{V}_{t} \boldsymbol{f}(i)=\left[\mathrm{e}^{t \boldsymbol{Q}} \boldsymbol{f}\right](i)-\int_{0}^{t} \mathrm{e}^{(t-s) \boldsymbol{Q}} \psi_{i}\left(V_{s} \boldsymbol{f}\right) \mathrm{d} s
$$

where $\boldsymbol{f}=(f(1), \cdots, f(d))^{T} \in \mathbb{R}_{+}^{d}$ and $\boldsymbol{Q}$ is the infinitesimal generator of the associated Markov chain. Note that a straightforward manipulation in the spirit of Theorem 3.1.2 of Dynkin (2002) implies that

$$
\begin{aligned}
\boldsymbol{V}_{t} \boldsymbol{f}(i) & =f(i)+\int_{0}^{t}\left[\boldsymbol{Q} V_{s} \boldsymbol{f}\right](i)-\int_{0}^{t} \psi_{i}\left(V_{s} \boldsymbol{f}\right) \mathrm{d} s \\
& =f(i)+\int_{0}^{t}\left\langle V_{s} \boldsymbol{f}, \boldsymbol{Q}^{T} \boldsymbol{e}_{i}\right\rangle-\int_{0}^{t} \psi_{i}\left(V_{s} \boldsymbol{f}\right) \mathrm{d} s,
\end{aligned}
$$

where $\boldsymbol{Q}^{T}$ is the transpose matrix of $\boldsymbol{Q}$. In turn, we note that this is equivalent to the unique semigroup evolution that solves (1.2), albeit that the branching mechanism

$$
\widetilde{\psi}_{i}(\boldsymbol{u}):=-\left\langle\boldsymbol{u}, \boldsymbol{Q}^{T} \boldsymbol{e}_{i}\right\rangle+\psi_{i}(\boldsymbol{u}) \quad \boldsymbol{u} \in \mathbb{R}_{+}^{d}, i \in E .
$$

We therefore suppose in the remaining part of this paper that $\left\{\boldsymbol{X}_{t}, t \geq 0\right\}$ is a $\psi$-MCSBP. Denote by $\boldsymbol{M}(t):=\left(M(t)_{i, j}\right)_{d \times d}$ the matrix with elements

$$
M(t)_{i, j}:=\mathbb{E}_{\boldsymbol{e}_{i}}\left[\left\langle\boldsymbol{e}_{j}, \boldsymbol{X}_{t}\right\rangle\right], \quad i, j \in E, t \geq 0 .
$$

By Barczy et al. (2015, Lemma 3.4) we have

$$
\boldsymbol{M}(t)=\mathrm{e}^{t \boldsymbol{B}^{T}}, \quad t \geq 0
$$

where $\boldsymbol{B}^{T}$ is the transpose of $\boldsymbol{B}$. Observe that for any initial vector $\boldsymbol{x}_{0}$ and any $\boldsymbol{x} \in \mathbb{R}_{+}^{d}$,

$$
\mathbb{E}_{\boldsymbol{x}_{0}}\left[\left\langle\boldsymbol{x}, \boldsymbol{X}_{t}\right\rangle\right]=\boldsymbol{x}_{0}^{T} \boldsymbol{M}(t) \boldsymbol{x}, \quad t \geq 0 .
$$

Moreover, by (1.3) and the Itô calculus, for all $\boldsymbol{x} \in \mathbb{R}^{d}$ and $s \leq r \leq t$, we obtain

$$
\begin{aligned}
\left\langle\boldsymbol{M}(t-r) \boldsymbol{x}, \boldsymbol{X}_{r}\right\rangle & =\left\langle\boldsymbol{M}(t-s) \boldsymbol{x}, \boldsymbol{X}_{s}\right\rangle+\sum_{i \in E} \int_{s}^{r}[\boldsymbol{M}(t-u) \boldsymbol{x}]_{i} \sqrt{2 c_{i} X_{u, i}} \mathrm{~d} W_{u, i} \\
& +\sum_{i \in E} \int_{s}^{r} \int_{\mathbb{R}_{+}^{d}} \int_{0}^{\infty}\langle\boldsymbol{M}(t-u) \boldsymbol{x}, \boldsymbol{z}\rangle \mathbf{1}_{\left\{l \leq X_{u-, i}\right\}} \widetilde{N}_{i}(\mathrm{~d} u, \mathrm{~d} \boldsymbol{z}, \mathrm{d} l) .
\end{aligned}
$$


In particular, if we take $r=t$, we get the following equation

$$
\begin{aligned}
\left\langle\boldsymbol{x}, \boldsymbol{X}_{t}\right\rangle=\langle\boldsymbol{M}(t-s) & \left.\boldsymbol{x}, \boldsymbol{X}_{s}\right\rangle+\sum_{i \in E} \int_{s}^{t}[\boldsymbol{M}(t-u) \boldsymbol{x}]_{i} \sqrt{2 c_{i} X_{u, i}} \mathrm{~d} W_{u, i} \\
& +\sum_{i \in E} \int_{s}^{t} \int_{\mathbb{R}_{+}^{d}} \int_{0}^{\infty}\langle\boldsymbol{M}(t-u) \boldsymbol{x}, \boldsymbol{z}\rangle \mathbf{1}_{\left\{l \leq X_{u-, i}\right\}} \widetilde{N}_{i}(\mathrm{~d} u, \mathrm{~d} \boldsymbol{z}, \mathrm{d} l) .
\end{aligned}
$$

A vector $\boldsymbol{u} \in \mathbb{R}^{d}$ is called a $\lambda$-right (resp. left) eigenvector if for all $t \geq 0$,

$$
\boldsymbol{M}(t) \boldsymbol{u}=\mathrm{e}^{\lambda t} \boldsymbol{u}, \quad\left(\operatorname{resp} . \boldsymbol{u}^{T} \boldsymbol{M}(t)=\mathrm{e}^{\lambda t} \boldsymbol{u}^{T}\right) .
$$

If $\boldsymbol{u}$ is a $\lambda$-right eigenvector, define

$$
W_{t}^{\lambda}(\boldsymbol{u}):=\mathrm{e}^{-\lambda t}\left\langle\boldsymbol{u}, \boldsymbol{X}_{t}\right\rangle, \quad t \geq 0 .
$$

Then it was shown in Proposition 3 of Kyprianou and Palau (2018+) that if $\boldsymbol{u}$ is a $\lambda$-right eigenvector with $\lambda \in \mathbb{R}$, then for any $\boldsymbol{x} \in \mathbb{R}_{+}^{d},\left\{W_{t}^{\lambda}(\boldsymbol{u}), t \geq 0\right\}$ is a martingale under $\mathbb{P}_{\boldsymbol{x}}$.

Suppose that $\boldsymbol{M}(t)$ is irreducible (exists $t_{0}>0$ such that $M\left(t_{0}\right)_{i, j}>0$ for all $i, j \in E)$. The Perron-Frobenius theory implies that there exist $\lambda_{1} \in \mathbb{R}$ and right and left associated eigenvectors $\phi, \widehat{\phi} \in \mathbb{R}_{+}^{d}$ with all coordinates strictly positive such that $\boldsymbol{M}(t) \boldsymbol{\phi}=\mathrm{e}^{\lambda_{1} t} \boldsymbol{\phi}$ and $\widehat{\boldsymbol{\phi}}^{T} \boldsymbol{M}(t)=\mathrm{e}^{\lambda_{1} t} \widehat{\boldsymbol{\phi}}^{T}$, for all $t \geq 0$. We note from (1.6) that this is equivalent to the statement that $\phi$ and $\widehat{\phi}$ are right and left eigenvectors for $\boldsymbol{B}^{T}$ with common eigenvalue $\lambda_{1}$. For convenience we shall normalise $\boldsymbol{\phi}$ and $\widehat{\phi}$ such that $\langle\boldsymbol{\phi}, \mathbf{1}\rangle=1=\langle\boldsymbol{\phi}, \widehat{\phi}\rangle$. Moreover, any other eigenvalue $\lambda$ satisfies $\lambda_{1}>\Re(\lambda)$ and

$$
\lim _{t \rightarrow \infty} \boldsymbol{M}(t) \mathrm{e}^{-\lambda_{1} t}=\boldsymbol{P}:=\left(\boldsymbol{\phi}_{i} \widehat{\boldsymbol{\phi}}_{j}\right)_{i, j \in E \times E}
$$

In addition, Barczy and Pap (2016, Lemma A.3) proved that there exist $c_{1}, c_{2}, c_{3}>$ 0 such that

$$
\left\|\boldsymbol{M}(t) \mathrm{e}^{-\lambda_{1} t}-\boldsymbol{P}\right\| \leq c_{1} \mathrm{e}^{-c_{2} t} \quad \text { and } \quad\|\boldsymbol{M}(t)\| \leq c_{3} \mathrm{e}^{\lambda_{1} t} \quad \text { for all } t \in \mathbb{R}_{+} .
$$

In order to simplify notation, we will denote by

$$
W_{t}:=W_{t}^{\lambda_{1}}(\phi)=\mathrm{e}^{-\lambda_{1} t}\left\langle\phi, \boldsymbol{X}_{t}\right\rangle, \quad t \geq 0 .
$$

Observe that $W_{t}$ is a non-negative martingale and has a limit a.s. that we will denote by $W_{\infty}$.

We say that the $\psi$-MCSBP is subcritical, critical, or supercritical according as $\lambda_{1}<0, \lambda_{1}=0$, or $\lambda_{1}>0$. This classification is consistent with the corresponding classification for single-type continuous state branching processes, see, e.g., Li (2011, Page 58). With the use of (1.10), it was proved in Kyprianou and Palau (2018+), that in the subcritical and critical cases the process has extinction a.s. In this paper, we want to find the asymptotic behaviour in the supercritical case.

The following Theorem 1.3 gives a relationship between the $\mathbb{L}^{1}$-convergence of the martingale $\left\{W_{t}, t \geq 0\right\}$ and the following condition:

$$
\sum_{i \in E} \int_{1 \leq\langle\mathbf{1}, \boldsymbol{z}\rangle<\infty}\langle\mathbf{1}, \boldsymbol{z}\rangle \ln (\langle\mathbf{1}, \boldsymbol{z}\rangle) \mu_{i}(\mathrm{~d} \boldsymbol{z})<\infty . \quad(x \log x \text { condition })
$$

If $\mu_{i}(\mathrm{~d} \boldsymbol{z})=\boldsymbol{\pi} \Pi_{i}(\mathrm{~d} r)$, where $\boldsymbol{z}=r \boldsymbol{\pi}$ with $\Pi_{i}$ being a measure on $(0, \infty)$ and $\boldsymbol{\pi}$ a fixed probability mass function on the type space $E$ in vector form, the following 
theorem comes from Theorem 5.1 and Theorem 6.2 in Ren et al. (2016). See also Kyprianou and Palau (2018+, Theorem 6).

Theorem 1.3. Suppose that $\lambda_{1}>0$. The following assertions hold:

(1) If ( $x \log x$ condition) holds, then for any $\mathbb{P}_{\boldsymbol{x}}, W_{\infty}$ is the $\mathbb{L}^{1}\left(\mathbb{P}_{\boldsymbol{x}}\right)$ limit of $W_{t}$ as $t \rightarrow \infty$.

(2) If ( $x \log x$ condition) doesn't hold, then for any $\boldsymbol{x} \in \mathbb{R}_{+}^{d}, W_{\infty}=0, \mathbb{P}_{\boldsymbol{x}}$ a.s.

We will prove this theorem in Section 2. By using this theorem we obtain a strong law of large numbers for MCSBPs. This result matches perfectly classical analogues for multi-type Galton-Watson processes; see for example Theorem V.6.1 of Athreya and Ney (2004).

Theorem 1.4. Suppose that $\boldsymbol{X}$ is a MCSBP with principal eigenvalue $\lambda_{1}>0$ and right and left associated eigenvectors $\boldsymbol{\phi}, \widehat{\boldsymbol{\phi}} \in \mathbb{R}_{+}^{d}$. Then for any $\boldsymbol{x} \in \mathbb{R}_{+}^{d}$,

$$
\lim _{t \rightarrow \infty} \mathrm{e}^{-\lambda_{1} t} \boldsymbol{X}_{t}=W_{\infty} \widehat{\boldsymbol{\phi}}, \quad \mathbb{P}_{\boldsymbol{x}} \text { a.s. }
$$

As a corollary we obtain the following convergence on rates of types.

Theorem 1.5. For any $\boldsymbol{x} \in \mathbb{R}_{+}^{d} \backslash\{\boldsymbol{0}\}$ we have, conditional on non-extinction,

$$
\lim _{t \rightarrow \infty} \frac{\boldsymbol{X}_{t}}{\left\langle\mathbf{1}, \boldsymbol{X}_{t}\right\rangle}=\frac{\widehat{\phi}}{\langle\mathbf{1}, \widehat{\phi}\rangle}, \quad \mathbb{P}_{\boldsymbol{x}} \text { a.s. }
$$

The remainder of this paper is structured as follows. In Section 2 we prove Theorem 1. The proof of Theorem 2 is given in Section 3.

\section{Spine decomposition}

A now classical way to prove Theorem 2 is to find a spine decomposition for $\left\{\boldsymbol{X}_{t}, t \geq 0\right\}$ under the Doob $h$-transform associated with $W$. More precisely, for any $\boldsymbol{x} \in \mathbb{R}_{+}^{d}$, using the martingale $\left(W_{t}, t \geq 0\right)$ we define a new probability measure via

$$
\left.\frac{\mathrm{d} \widetilde{\mathbb{P}}_{\boldsymbol{x}}}{\mathrm{d} \mathbb{P}_{\boldsymbol{x}}}\right|_{\mathcal{F}_{t}}=\frac{1}{\langle\boldsymbol{\phi}, \boldsymbol{x}\rangle} W_{t}, \quad t \geq 0 .
$$

Where $\left\{\mathcal{F}_{t}, t \geq 0\right\}$ is the natural filtration generated by $\boldsymbol{X}$.

Let $\left(\eta_{t}, t \geq 0\right)$ be a Markov chain on $E$ with infinitesimal generator $\boldsymbol{L}$, a $d \times d$ matrix defined by

$$
L_{i j}=\frac{1}{\phi(i)}\left(B_{i j}^{T}-\mathbf{1}_{\{i=j\}} \lambda_{1}\right) \phi(j), \quad i, j \in E .
$$

Denote by $\left(\boldsymbol{P}_{i}^{\phi}, i \in E\right)$ the probabilities of $\eta$ such that $\boldsymbol{P}_{i}^{\phi}\left(\eta_{0}=i\right)=1$ for all $i \in \mathbb{E}$.

Theorem 2.1. If $\boldsymbol{X}$ is a MCSBP, then for any $\boldsymbol{x} \in \mathbb{R}_{+}^{d}$ and $\boldsymbol{f} \in \mathbb{R}_{+}^{d}$,

$$
\begin{gathered}
\widetilde{\mathbb{E}}_{\boldsymbol{x}}\left[\mathrm{e}^{-\left\langle\boldsymbol{f}, \boldsymbol{X}_{t}\right\rangle}\right]=\mathbb{E}_{\boldsymbol{x}}\left[\mathrm{e}^{\left.-\left\langle\boldsymbol{f}, \boldsymbol{X}_{t}\right\rangle\right] \times}\right. \\
\boldsymbol{E}_{\boldsymbol{\phi} \boldsymbol{x}}^{\boldsymbol{\phi}}\left[\exp \left\{-\int_{0}^{t}\left(2 c\left(\eta_{s}\right) v_{\eta_{s}}(t-s, \boldsymbol{f})+\int_{\mathbb{R}_{+}^{d}} z\left(\eta_{s}\right)\left(1-\mathrm{e}^{-\langle\boldsymbol{v}(t-s, \boldsymbol{f}), \boldsymbol{z}\rangle}\right) \mu_{\eta_{s}}(\mathrm{~d} \boldsymbol{z})\right) \mathrm{d} s\right\} \times\right. \\
\left.\prod_{s \leq t} \mathcal{A}_{\eta_{s-}, \eta_{s}}^{t-s}\right],
\end{gathered}
$$


where the matrices $\left\{\mathcal{A}^{s}: s \geq 0\right\}$ are given by

$$
\mathcal{A}_{i, j}^{s}=\left(\mathbf{1}_{\{i \neq j\}} \frac{1}{B_{i j}^{T}} \int_{\mathbb{R}_{+}^{d}} z(j)\left(\mathrm{e}^{-\langle\boldsymbol{v}(s, \boldsymbol{f}), \boldsymbol{z}\rangle}-1\right) \mu_{i}(\mathrm{~d} \boldsymbol{z})+1\right), \quad i, j \in E,
$$

and

$$
\boldsymbol{P}_{\phi \boldsymbol{x}}^{\boldsymbol{\phi}}(\cdot)=\sum_{i \in E} \frac{\phi(i) x(i)}{\langle\boldsymbol{\phi}, \boldsymbol{x}\rangle} \boldsymbol{P}_{i}^{\boldsymbol{\phi}}(\cdot)
$$

with associated expectation operator $\boldsymbol{E}_{\boldsymbol{\phi} \boldsymbol{x}}^{\phi}(\cdot)$.

Proof: We start by noting that

$$
\widetilde{\mathbb{E}}_{\boldsymbol{x}}\left[\mathrm{e}^{-\left\langle\boldsymbol{f}, \boldsymbol{X}_{t}\right\rangle}\right]=\frac{\mathrm{e}^{-\lambda_{1} t}}{\langle\boldsymbol{\phi}, \boldsymbol{x}\rangle} \mathbb{E}_{\boldsymbol{x}}\left[\left\langle\boldsymbol{\phi}, \boldsymbol{X}_{t}\right\rangle \mathrm{e}^{-\left\langle\boldsymbol{f}, \boldsymbol{X}_{t}\right\rangle}\right] .
$$

Replacing $\boldsymbol{f}$ by $\boldsymbol{f}+\lambda \boldsymbol{\phi}$ in (1.1) and (1.2) and differentiating with respect to $\lambda$ and then setting $\lambda=0$, we obtain

$$
\begin{aligned}
\widetilde{\mathbb{E}}_{\boldsymbol{x}}\left[\mathrm{e}^{\left.-\left\langle\boldsymbol{f}, \boldsymbol{X}_{t}\right\rangle\right]}\right. & =\mathbb{E}_{\boldsymbol{x}}\left[\mathrm{e}^{-\left\langle\boldsymbol{f}, \boldsymbol{X}_{t}\right\rangle}\right] \sum_{i \in E} \frac{\phi(i) x(i)}{\langle\boldsymbol{\phi}, \boldsymbol{x}\rangle} \theta^{t}(i) \\
& =\mathbb{E}_{\boldsymbol{x}}\left[\mathrm{e}^{-\left\langle\boldsymbol{f}, \boldsymbol{X}_{t}\right\rangle}\right] \frac{\left\langle\theta^{t}, \boldsymbol{\phi} \circ \boldsymbol{x}\right\rangle}{\langle\boldsymbol{\phi}, \boldsymbol{x}\rangle},
\end{aligned}
$$

where $\circ$ denotes element wise multiplication of vectors and, for $t \geq 0, \theta^{t}$ is the vector with entries

$$
\theta^{t}(i):=\left.\frac{1}{\phi(i)} \mathrm{e}^{-\lambda_{1} t} \frac{\partial}{\partial \lambda} v_{i}(t, \boldsymbol{f}+\lambda \boldsymbol{\phi})\right|_{\lambda=0} .
$$

By an integration by parts, using (1.2) and (1.4) and that $\left(\boldsymbol{B}_{i}\right)_{j}=\boldsymbol{B}_{i j}^{T}$, we get that $\theta^{t}(i)$ is also the unique vector solution to

$$
\begin{gathered}
\theta^{t}(i)=1+\int_{0}^{t} \frac{1}{\phi(i)}\left[\left(B^{T}-\lambda_{1} I\right)\left(\boldsymbol{\phi} \circ \theta^{s}\right)\right](i) \mathrm{d} s-\int_{0}^{t} \theta^{s}(i) 2 c(i) v_{i}(s, \boldsymbol{f}) \mathrm{d} s \\
+\int_{0}^{t} \int_{\mathbb{R}_{+}^{d}}\left\langle\theta^{s}, \frac{\boldsymbol{\phi} \circ \boldsymbol{z}}{\phi(i)}\right\rangle\left(\mathrm{e}^{-\langle\boldsymbol{v}(s, \boldsymbol{f}), \boldsymbol{z}\rangle}-1\right) \mu_{i}(\mathrm{~d} \boldsymbol{z}) \mathrm{d} s .
\end{gathered}
$$

Recall the definition of $\boldsymbol{L}$ and note that it conforms to the definition of an intensity matrix of a Markov chain, thanks to the fact that $\phi$ is an eigenvector of $\boldsymbol{B}^{T}$. A (vectorial) integration by parts in the spirit of Theorem 3.1.2 of Dynkin (2002),

$$
\begin{aligned}
& {\left[\theta^{t}\right](i)=\left[\mathrm{e}^{t \boldsymbol{L}} \mathbf{1}\right](i) }-\int_{0}^{t} \mathrm{e}^{(t-s) \boldsymbol{L}}\left[\theta^{s}(\cdot) \circ(2 c(\cdot) v \cdot(s, \boldsymbol{f}))\right](i) \mathrm{d} s \\
&+\int_{0}^{t} \mathrm{e}^{(t-s) \boldsymbol{L}}\left[\int_{\mathbb{R}_{+}^{d}}\left\langle\theta^{s}, \boldsymbol{\phi} \circ \boldsymbol{z}\right\rangle\left(\mathrm{e}^{-\langle\boldsymbol{v}(s, \boldsymbol{f}), \boldsymbol{z}\rangle}-1\right) \frac{\mu \cdot(\mathrm{d} \boldsymbol{z})}{\phi(\cdot)}\right](i) \mathrm{d} s,
\end{aligned}
$$

where $\mathbf{1}=(1, \cdots, 1)^{T} \in \mathbb{R}_{+}^{d}$. Then appealing to the fact that $\left\{\mathrm{e}^{t \boldsymbol{L}}: t \geq 0\right\}$ is the semigroup of $\left(\eta, \boldsymbol{P}_{i}^{\phi}\right), i \in E$,

$$
\begin{aligned}
\theta^{t}(i)= & \left.\boldsymbol{E}_{i}^{\phi}[\mathbf{1}]-\int_{0}^{t} \boldsymbol{E}_{i}^{\phi}\left[2 \theta^{s}\left(\eta_{t-s}\right) c\left(\eta_{t-s}\right) v_{\eta_{t-s}}(s, \boldsymbol{f})\right)\right] \mathrm{d} s \\
& +\int_{0}^{t} \boldsymbol{E}_{i}^{\phi}\left[\int_{\mathbb{R}_{+}^{d}}\left\langle\theta^{s}, \boldsymbol{\phi} \circ \boldsymbol{z}\right\rangle\left(\mathrm{e}^{-\langle\boldsymbol{v}(s, \boldsymbol{f}), \boldsymbol{z}\rangle}-1\right) \frac{\mu_{\eta_{t-s}}(\mathrm{~d} \boldsymbol{z})}{\phi\left(\eta_{t-s}\right)}\right] \mathrm{d} s .
\end{aligned}
$$


Next, we make a change of variable $u=t-s$ and separate the last inner product into two parts. For all $s \leq t$ and $\boldsymbol{z} \in \mathbb{R}_{+}^{d}$

$$
\left\langle\theta^{t-s}, \boldsymbol{\phi} \circ \boldsymbol{z}\right\rangle=\theta^{t-s}\left(\eta_{s}\right) \phi\left(\eta_{s}\right) z\left(\eta_{s}\right)+\sum_{j \neq \eta_{s}} \theta^{t-s}(j) \phi(j) z(j) .
$$

Therefore, (2.2) is transformed into

$$
\begin{aligned}
\theta^{t}(i)=\boldsymbol{E}_{i}^{\phi} & {\left.\left[g\left(\eta_{t}\right)\right]-\int_{0}^{t} \boldsymbol{E}_{i}^{\phi}\left[2 \theta^{t-s}\left(\eta_{s}\right) c\left(\eta_{s}\right) v_{\eta_{s}}(t-s, \boldsymbol{f})\right)\right] \mathrm{d} s } \\
+ & \int_{0}^{t} \boldsymbol{E}_{i}^{\phi}\left[\theta^{t-s}\left(\eta_{s}\right) \int_{\mathbb{R}_{+}^{d}} z\left(\eta_{s}\right)\left(\mathrm{e}^{-\langle\boldsymbol{v}(t-s, \boldsymbol{f}), \boldsymbol{z}\rangle}-1\right) \mu_{\eta_{s}}(\mathrm{~d} \boldsymbol{z})\right] \mathrm{d} s \\
+ & \int_{0}^{t} \boldsymbol{E}_{i}^{\phi}\left[\sum_{j \neq \eta_{s}} \theta^{t-s}(j) \frac{\phi(j)}{\phi\left(\eta_{s}\right)} \int_{\mathbb{R}_{+}^{d}} z(j)\left(\mathrm{e}^{-\langle\boldsymbol{v}(t-s, \boldsymbol{f}), \boldsymbol{z}\rangle}-1\right) \mu_{\eta_{s}}(\mathrm{~d} \boldsymbol{z})\right] \mathrm{d} s .
\end{aligned}
$$

Recall that (1.5) holds and hence, by applying Chen et al. (2018+, Lemma 6.1) to the Lévy system associated to $\boldsymbol{L}$, using, in their notation, the functions

$$
q(s, i)=2 c(i) v_{i}(s, \boldsymbol{f})+\int_{\mathbb{R}_{+}^{d}} z(i)\left(1-\mathrm{e}^{-\langle\boldsymbol{v}(s, \boldsymbol{f}), \boldsymbol{z}\rangle}\right) \mu_{i}(\mathrm{~d} \boldsymbol{z})
$$

and

$$
F(s, i, j)=\ln \left(\mathbf{1}_{\{i \neq j\}} \frac{1}{B_{j i}} \int_{\mathbb{R}_{+}^{d}} z(j)\left(\mathrm{e}^{-\langle\boldsymbol{v}(s, \boldsymbol{f}), \boldsymbol{z}\rangle}-1\right) \mu_{i}(\mathrm{~d} \boldsymbol{z})+1\right),
$$

we obtain

$$
\begin{gathered}
\theta^{t}(i)=\boldsymbol{E}_{i}^{\boldsymbol{\phi}}\left[\exp \left\{-\int_{0}^{t}\left(2 c\left(\eta_{s}\right) v_{\eta_{s}}(t-s, \boldsymbol{f})+\int_{\mathbb{R}_{+}^{d}} z\left(\eta_{s}\right)\left(1-\mathrm{e}^{-\langle\boldsymbol{v}(t-s, \boldsymbol{f}), \boldsymbol{z}\rangle}\right) \mu_{\eta_{s}}(\mathrm{~d} \boldsymbol{z})\right) \mathrm{d} s\right\}\right. \\
\left.\prod_{s \leq t} \mathcal{A}_{\eta_{s-}-\eta_{s}}^{t-s}\right]
\end{gathered}
$$

Theorem 2.1 suggests that the process $\left(\boldsymbol{X}_{t}, \widetilde{\mathbb{P}}_{\boldsymbol{x}}\right)$ is equal in law to a process $\left\{\boldsymbol{\Gamma}_{t}: t \geq 0\right\}$, whose law is henceforth denoted by $\mathrm{P}_{\boldsymbol{x}}, \boldsymbol{x} \in \mathbb{R}_{+}^{d}$, where

$$
\boldsymbol{\Gamma}_{t}=\boldsymbol{X}_{t}^{\prime}+\sum_{s \leq t: c} \boldsymbol{X}_{t-s}^{\mathrm{c}, s}+\sum_{s \leq t: \mathrm{d}} \boldsymbol{X}_{t-s}^{\mathrm{d}, s}+\sum_{s \leq t: \mathrm{j}} \boldsymbol{X}_{t-s}^{\mathrm{j}, s}, \quad t \geq 0
$$

such that $\boldsymbol{X}^{\prime}$ is an independent copy of $\left(\boldsymbol{X}_{t}, \mathbb{P}_{\boldsymbol{x}}\right)$ and the processes $\boldsymbol{X}^{\mathrm{c}, s}, \boldsymbol{X}^{\mathrm{d}, s}$ and $\boldsymbol{X}^{\mathrm{j}, s}$ are defined through a process of immigration as follows: Given the path of the Markov chain $\left(\eta, \boldsymbol{P}_{\phi \boldsymbol{x}}^{\phi}\right)$,

[continuous immigration] in a Poissonian way a $\psi$-MCSBP $\boldsymbol{X}^{\mathrm{c}, s}$ immigrates at $\left(s, \eta_{s}\right)$ with rate $\mathrm{d} s \times 2 c\left(\eta_{s}\right) \mathrm{d} \mathbf{N}_{\eta_{s}}$,

[discontinuous immigration] in a Poissonian way a $\psi$-MCSBP $\boldsymbol{X}^{\mathrm{d}, s}$ immigrates at $\left(s, \eta_{s}\right)$ with rate $\mathrm{d} s \times \int_{\mathbb{R}_{+}^{d}} z\left(\eta_{s}\right) \mu_{\eta_{s}}(\mathrm{~d} \boldsymbol{z}) \mathbb{P}_{\boldsymbol{z}}$ 
[jump immigration] at each jump time $s$ of $\eta$, a $\psi$-MCSBP $\boldsymbol{X}^{\mathrm{j}, s}$ immigrates at $\left(s, \eta_{s}\right)$ with law $\int_{\mathbb{R}_{+}^{d}} \nu_{\eta_{s-}, \eta_{s}}(\mathrm{~d} \boldsymbol{z}) \mathbb{P}_{\boldsymbol{z}}$, where for each $i, j \in E$,

$$
\nu_{i, j}(\mathrm{~d} \boldsymbol{z})=\mathbf{1}_{\{i \neq j\}} \frac{1}{B_{i j}^{T}} z(j) \mu_{i}(\mathrm{~d} \boldsymbol{z})+\left(1-\mathbf{1}_{\{i \neq j\}} \frac{1}{B_{i j}^{T}} \int_{\mathbb{R}_{+}^{d}} v(j) \mu_{i}(\mathrm{~d} \boldsymbol{v})\right) \delta_{\mathbf{0}}(\mathrm{d} \boldsymbol{z}) .
$$

Given $\eta$, the above three immigration processes are independent.

In the above description, the quantity $\mathbf{N}_{i}$ is the excursion measure of the $\psi$ MCSBP corresponding to $\mathbb{P}_{\boldsymbol{e}_{i}}$. To be more precise, Dynkin and Kuznetsov (2004) showed that associated to the laws $\left\{\mathbb{P}_{\boldsymbol{e}_{i}}: i \in E\right\}$ are the measures $\left\{\mathbf{N}_{i}: i \in E\right\}$, defined on the same measurable space, which satisfy

$$
\mathbf{N}_{i}\left(1-\mathrm{e}^{-\left\langle\boldsymbol{f}, \boldsymbol{X}_{t}\right\rangle}\right)=-\log \mathbb{E}_{\boldsymbol{e}_{i}}\left(\mathrm{e}^{-\left\langle\boldsymbol{f}, \boldsymbol{X}_{t}\right\rangle}\right),
$$

for all $t \geq 0$. A particular feature of $\mathbf{N}_{i}$ that we shall use later is that

$$
\mathbf{N}_{i}\left(\left\langle\boldsymbol{f}, \boldsymbol{X}_{t}\right\rangle\right)=\mathbb{E}_{\boldsymbol{e}_{i}}\left(\left\langle\boldsymbol{f}, \boldsymbol{X}_{t}\right\rangle\right) \text {. }
$$

Note, in order for the measures $\mathbf{N}_{i}, i=1, \cdots, d$, to be well defined, we need the assumption made in the introduction that $\mathbb{P}_{\boldsymbol{e}_{\boldsymbol{i}}}\left(\left\langle 1, X_{t}\right\rangle=0\right)>0$ for all $t>0$, $i=1, \cdots, d$.

Observe that the processes $\boldsymbol{X}^{\boldsymbol{c}}, \boldsymbol{X}^{\boldsymbol{d}}$ and $\boldsymbol{X}^{j}$ are initially zero valued, therefore, if $\boldsymbol{\Gamma}_{0}=\boldsymbol{x}$ then $\boldsymbol{X}_{0}^{\prime}=\boldsymbol{x}$. The following result corresponds to a classical spine decomposition, albeit now for the setting of an MCSBP. Note, we henceforth refer to the process $\eta$ as the spine. By following the same proof as Theorem 5 in Kyprianou and Palau (2018+) we can easily establish the next result.

Theorem 2.2 (Spine decomposition). For any $\boldsymbol{x} \in \mathbb{R}_{+}^{d},\left(\boldsymbol{\Gamma}_{t}, t \geq 0 ; \mathrm{P}_{\boldsymbol{x}}\right)$ is equal in law to $\left(\boldsymbol{X}_{t}, t \geq 0 ; \widetilde{\mathbb{P}}_{\boldsymbol{x}}\right)$.

For the sake of brevity, we leave the proof to the reader.

2.1. Proof of Theorem 1.3. We follow a well established line of reasoning. We know that $1 / W_{t}$ is a positive $\widetilde{\mathbb{P}}_{\boldsymbol{x}}$-supermartingale and hence $\lim _{t \rightarrow \infty} W_{t}$ exists $\widetilde{\mathbb{P}}_{\boldsymbol{x}}$-almost surely. Therefore $W_{t}$ converges in $\mathbb{L}^{1}\left(\mathbb{P}_{\boldsymbol{x}}\right)$ to a non-degenerated limit as soon as we prove that $\widetilde{\mathbb{P}}_{\boldsymbol{x}}\left(\liminf \operatorname{in}_{t \rightarrow \infty} W_{t}<\infty\right)=1$.

We consider the spine decomposition in Theorem 2.2. Given the spine $\eta$, let us write $\left(s, \boldsymbol{I}_{s}^{\mathrm{d}}, \boldsymbol{I}_{s}^{\mathrm{j}}\right)_{s \geq 0}$ for the process of immigrated vector along the spine (i.e. $\boldsymbol{I}_{s}^{\mathrm{d}}=$ $\boldsymbol{X}_{0}^{\mathrm{d}, s}$ and $\left.\boldsymbol{I}_{s}^{\mathrm{j}}=\boldsymbol{X}_{0}^{\mathrm{j}, s}\right)$. Then $\left(s, \boldsymbol{I}_{s}^{\mathrm{d}}\right)$ is Poissonian with intensity $\mathrm{d} s \times z\left(\eta_{s}\right) \mu_{\eta_{s}}(\mathrm{~d} \boldsymbol{z})$ and, if $s$ is such that $\eta_{s-} \neq \eta_{s}$ then $\boldsymbol{I}_{s}^{\mathrm{j}}$ is distributed according to $\nu_{\eta_{s-}, \eta_{s}}$. Let $\mathcal{S}=\sigma\left(\eta_{s},\left(s, \boldsymbol{I}_{s}^{\mathrm{d}}, \boldsymbol{I}_{s}^{\mathrm{j}}\right), s \geq 0\right)$ be the sigma algebra which informs the location of the spine and the vector issued at each immigration time and write

$$
Z_{t}:=\mathrm{e}^{-\lambda_{1} t}\left\langle\phi, \boldsymbol{\Gamma}_{t}\right\rangle
$$

Since $\left(\boldsymbol{\Gamma} ., \mathrm{P}_{\boldsymbol{x}}\right)$ is equal in law to $\left(\boldsymbol{X} ., \widetilde{\mathbb{P}}_{\boldsymbol{x}}\right)$, to prove $\widetilde{\mathbb{P}}_{\boldsymbol{x}}\left(\liminf _{t \rightarrow \infty} W_{t}<\infty\right)=1$, we only need to prove that

$$
\mathrm{P}_{\boldsymbol{x}}\left(\liminf _{t \rightarrow \infty} Z_{t}<\infty\right)=1 .
$$

By Fatou's Lemma

$$
\mathrm{E}_{\boldsymbol{x}}\left[\liminf _{t \rightarrow \infty} Z_{t} \mid \mathcal{S}\right] \leq \liminf _{t \rightarrow \infty} \mathrm{E}_{\boldsymbol{x}}\left[Z_{t} \mid \mathcal{S}\right] .
$$

It therefore remains to show that $\liminf _{t \rightarrow \infty} \mathrm{E}_{\boldsymbol{x}}\left[Z_{t} \mid \mathcal{S}\right]<\infty, \widetilde{\mathbb{P}}_{\boldsymbol{x}}$ a.s. 
By using that the processes $\boldsymbol{X}^{\mathrm{c}, s}, \boldsymbol{X}^{\mathrm{d}, s}$ conditioned on $\mathcal{S}$ are Poissonian, the description of $\boldsymbol{X}^{\mathrm{j}, s}, \boldsymbol{I}^{\mathrm{j}}$. and $\boldsymbol{I}^{\mathrm{d}}$, formula (2.3) and that $W$ is a martingale, we have that for $t \geq 0$ and $\boldsymbol{x} \in \mathbb{R}_{+}^{d}$,

$$
\begin{aligned}
& \liminf _{t \rightarrow \infty} \mathrm{E}_{\boldsymbol{x}}\left[Z_{t} \mid \mathcal{S}\right] \\
& =\langle\boldsymbol{\phi}, \boldsymbol{x}\rangle+\int_{0}^{\infty} 2 c\left(\eta_{s}\right) \mathrm{e}^{-\lambda_{1} s} \phi\left(\eta_{s}\right) \mathrm{d} s+\sum_{0 \leq s} \mathrm{e}^{-\lambda_{1} s}\left\langle\boldsymbol{\phi}, \boldsymbol{I}_{s}^{\mathrm{d}}\right\rangle+\sum_{0 \leq s} \mathrm{e}^{-\lambda_{1} s}\left\langle\boldsymbol{\phi}, \boldsymbol{I}_{s}^{\mathrm{j}}\right\rangle .
\end{aligned}
$$

Since $\lambda_{1}>0$, the first integral is finite. We need to prove that the other sums are finite a.s. In order to do it, we will decompose the sum in small jumps: $\left\{\left(s, \boldsymbol{I}_{s}^{\mathrm{d}}\right)\right.$ : $\left.\left\langle\mathbf{1}, \boldsymbol{I}_{s}^{\mathrm{d}}\right\rangle \leq \mathrm{e}^{\lambda_{1} s}\right\}$ and big jumps: $\left\{\left(s, \boldsymbol{I}_{s}^{\mathrm{d}}\right):\left\langle\mathbf{1}, \boldsymbol{I}_{s}^{\mathrm{d}}\right\rangle>\mathrm{e}^{\lambda_{1} s}\right\}$, and we handle $\boldsymbol{I}_{s}^{\mathrm{j}}$ in similar way. Denote $M_{\phi}:=\max \{\phi(i): i \in E\}$. For the small jumps, by the definition of $\nu$,

$$
\begin{aligned}
& \mathrm{E}_{\boldsymbol{x}}\left[\sum_{0 \leq s} \mathrm{e}^{-\lambda_{1} s}\left\langle\boldsymbol{\phi}, \boldsymbol{I}_{s}^{\mathrm{d}}\right\rangle \mathbf{1}_{\left\{\left\langle\mathbf{1}, \boldsymbol{I}_{s}^{\mathrm{d}}\right\rangle \leq \mathrm{e}^{\lambda_{1} s}\right\}}+\sum_{0 \leq s} \mathrm{e}^{-\lambda_{1} s}\left\langle\boldsymbol{\phi}, \boldsymbol{I}_{s}^{\mathrm{j}}\right\rangle \mathbf{1}_{\left\{\left\langle\mathbf{1}, \boldsymbol{I}_{s}^{\mathrm{j}}\right\rangle \leq \mathrm{e}^{\lambda_{1} s}\right\}}\right] \\
& =\mathrm{E}_{\boldsymbol{x}}\left[\int_{0}^{\infty} \int_{\langle\mathbf{1}, \boldsymbol{z}\rangle \leq \mathrm{e}^{\lambda_{1} s}} \mathrm{e}^{-\lambda_{1} s} z\left(\eta_{s}\right)\langle\boldsymbol{\phi}, \boldsymbol{z}\rangle \mu_{\eta_{s}}(\mathrm{~d} \boldsymbol{z}) \mathrm{d} s\right. \\
& \left.+\int_{0}^{\infty} \int_{\langle\mathbf{1}, \boldsymbol{z}\rangle \leq \mathrm{e}^{\lambda_{1} s}} \mathrm{e}^{-\lambda_{1} s}\langle\boldsymbol{\phi}, \boldsymbol{z}\rangle \nu_{\eta_{s-}, \eta_{s}}(\mathrm{~d} \boldsymbol{z}) \mathrm{d} s\right] \\
& \leq C M_{\phi} \sum_{i \in E} \int_{0}^{\infty} \int_{\langle\mathbf{1}, \boldsymbol{z}\rangle \leq \mathrm{e}^{\lambda_{1} s}} \mathrm{e}^{-\lambda_{1} s}\langle\mathbf{1}, \boldsymbol{z}\rangle^{2} \mu_{i}(\mathrm{~d} \boldsymbol{z}) \mathrm{d} s
\end{aligned}
$$

where $C$ is a positive constant. For each $i \in E$,

$$
\begin{aligned}
& \int_{0}^{\infty} \int_{\langle\mathbf{1}, \boldsymbol{z}\rangle \leq \mathrm{e}^{\lambda_{1} s}} \mathrm{e}^{-\lambda_{1} s}\langle\mathbf{1}, \boldsymbol{z}\rangle^{2} \mu_{i}(\mathrm{~d} \boldsymbol{z}) \mathrm{d} s \\
& \leq \int_{\langle\mathbf{1}, \boldsymbol{z}\rangle \leq 1}\langle\mathbf{1}, \boldsymbol{z}\rangle^{2} \mu_{i}(\mathrm{~d} \boldsymbol{z})+\int_{\langle\mathbf{1}, \boldsymbol{z}\rangle>1}\langle\mathbf{1}, \boldsymbol{z}\rangle \mu_{i}(\mathrm{~d} \boldsymbol{z})<\infty
\end{aligned}
$$

Therefore,

$$
\sum_{0 \leq s} \mathrm{e}^{-\lambda_{1} s}\left\langle\boldsymbol{\phi}, \boldsymbol{I}_{s}^{\mathrm{d}}\right\rangle \mathbf{1}_{\left\{\left\langle\mathbf{1}, \boldsymbol{I}_{s}^{\mathrm{d}}\right\rangle \leq \mathrm{e}^{\lambda_{1} s}\right\}}+\sum_{0 \leq s} \mathrm{e}^{-\lambda_{1} s}\left\langle\boldsymbol{\phi}, \boldsymbol{I}_{s}^{\mathrm{j}}\right\rangle \mathbf{1}_{\left\{\left\langle\mathbf{1}, \boldsymbol{I}_{s}^{\mathrm{j}}\right\rangle \leq \mathrm{e}^{\lambda_{1} s}\right\}}<\infty, \quad \mathrm{P}_{\boldsymbol{x}} \text { a.s. }
$$

For the big jumps, using Fubini's Theorem, we get

$$
\begin{aligned}
& \mathrm{E}_{\boldsymbol{x}}\left[\sum_{0 \leq s} \mathbf{1}_{\left\{\left\langle\mathbf{1}, \boldsymbol{I}_{s}^{\mathrm{d}}\right\rangle>\mathrm{e}^{\lambda_{1} s}\right\}}+\sum_{0 \leq s} \mathbf{1}_{\left\{\left\langle\mathbf{1}, \mathbf{I}_{s}^{\mathrm{j}}\right\rangle>\mathrm{e}^{\lambda_{1} s}\right\}}\right] \\
= & \mathrm{E}_{\boldsymbol{x}}\left[\int_{0}^{\infty} \int_{\langle\mathbf{1}, \boldsymbol{z}\rangle>\mathrm{e}^{\lambda_{1} s}} z\left(\eta_{s}\right) \mu_{\eta_{s}}(\mathrm{~d} \boldsymbol{z}) \mathrm{d} s+\int_{0}^{\infty} \int_{\langle\mathbf{1}, \boldsymbol{z}\rangle>\mathrm{e}^{\lambda_{1} s}} \nu_{\eta_{s-}, \eta_{s}}(\mathrm{~d} \boldsymbol{z}) \mathrm{d} s\right] \\
\leq & C \sum_{i \in E} \int_{0}^{\infty} \int_{\langle\mathbf{1}, \boldsymbol{z}\rangle>\mathrm{e}^{\lambda_{1} s}}\langle\mathbf{1}, \boldsymbol{z}\rangle \mu_{i}(\mathrm{~d} \boldsymbol{z}) \mathrm{d} s \\
= & \frac{C}{\lambda_{1}} \sum_{i \in E} \int_{\langle\mathbf{1}, \boldsymbol{z}\rangle>1}^{\langle\mathbf{1}, \boldsymbol{z}\rangle \ln (\langle\mathbf{1}, \boldsymbol{z}\rangle) \mu_{i}(\mathrm{~d} \boldsymbol{z})<\infty}
\end{aligned}
$$


This implies that $\mathrm{P}_{\boldsymbol{x}}$ a.s. we have finitely many big jumps and therefore

$$
\sum_{0 \leq s} \mathrm{e}^{-\lambda_{1} s}\left\langle\boldsymbol{\phi}, \boldsymbol{I}_{s}^{\mathrm{d}}\right\rangle \mathbf{1}_{\left\{\left\langle\mathbf{1}, \boldsymbol{I}_{s}^{\mathrm{d}}\right\rangle>\mathrm{e}^{\lambda_{1} s}\right\}}+\sum_{0 \leq s} \mathrm{e}^{-\lambda_{1} s}\left\langle\boldsymbol{\phi}, \boldsymbol{I}_{s}^{\mathrm{j}}\right\rangle \mathbf{1}_{\left\{\left\langle\mathbf{1}, \boldsymbol{I}_{s}^{\mathrm{j}}\right\rangle>\mathrm{e}^{\lambda_{1} s}\right\}}<\infty, \quad \mathrm{P}_{\boldsymbol{x}} \text { a.s. }
$$

So, $\liminf _{t \rightarrow \infty} \mathrm{E}_{\boldsymbol{x}}\left[Z_{t} \mid \mathcal{S}\right]<\infty, \mathrm{P}_{\boldsymbol{x}}$ a.s.

Now, we will prove the second part of the Theorem. Since

$$
\widetilde{\mathbb{P}}_{\boldsymbol{x}}\left(\limsup _{t \rightarrow \infty} W_{t}=\infty\right)=\mathrm{P}_{\boldsymbol{x}}\left(\limsup _{t \rightarrow \infty} Z_{t}=\infty\right)
$$

if we prove that

$$
\limsup _{t \rightarrow \infty} Z_{t}=\infty \quad \mathrm{P}_{\boldsymbol{x}} \text { a.s. }
$$

then $\widetilde{\mathbb{P}}_{\boldsymbol{x}}$ and $\mathbb{P}_{\boldsymbol{x}}$ are singular and hence

$$
\mathbb{P}_{\boldsymbol{x}}\left(\limsup _{t \rightarrow \infty} W_{t}=0\right)=1
$$

It remains to show (2.5). Suppose that for a fixed $i \in E$,

$$
\int_{\langle\mathbf{1}, \boldsymbol{z}\rangle>1}\langle\mathbf{1}, \boldsymbol{z}\rangle \ln (\langle\mathbf{1}, \boldsymbol{z}\rangle) \mu_{i}(\mathrm{~d} \boldsymbol{z})=\infty .
$$

We will divide the proof in two parts.

(i) First assume that

$$
\int_{\langle\mathbf{1}, \boldsymbol{z}\rangle>1} z(i) \ln (\langle\mathbf{1}, \boldsymbol{z}\rangle) \mu_{i}(\mathrm{~d} \boldsymbol{z})=\infty .
$$

Denote by $\mathcal{T}$ the set of times at which we immigrate $\left(s, \boldsymbol{X}^{d, s}\right)$ along the spine, then for $s \in \mathcal{T}$,

$$
Z_{s} \geq \mathrm{e}^{-\lambda_{1} s}\left\langle\boldsymbol{\phi}, \boldsymbol{X}_{0}^{d, s}\right\rangle
$$

To prove $(2.5)$, we only need to prove that

$$
\limsup _{\mathcal{T} \ni s \rightarrow \infty} \mathrm{e}^{-\lambda_{1} s}\left\langle\boldsymbol{\phi}, \boldsymbol{X}_{0}^{d, s}\right\rangle=\infty \text {. }
$$

Since $\mathrm{e}^{-\lambda_{1} s}\left\langle\boldsymbol{\phi}, \boldsymbol{X}_{0}^{d, s}\right\rangle \geq m_{\boldsymbol{\phi}} \mathrm{e}^{-\lambda_{1} s}\left\langle\mathbf{1}, \boldsymbol{X}_{0}^{d, s}\right\rangle$, where $m_{\boldsymbol{\phi}}:=\min \{\phi(i): i \in E\}$, we only need to prove that

$$
\limsup _{\mathcal{T} \ni s \rightarrow \infty} \mathrm{e}^{-\lambda_{1} s}\left\langle\mathbf{1}, \boldsymbol{X}_{0}^{d, s}\right\rangle=\infty .
$$

For $T, K>1$, define the subsets

$$
A_{T, K}:=\sharp\left\{s \in \mathcal{T} \cap(T, \infty):\left\langle\mathbf{1}, \boldsymbol{X}_{0}^{d, s}\right\rangle>K \mathrm{e}^{\lambda_{1} s}\right\} .
$$

Then, given the Markov chain $\left(\eta, \boldsymbol{P}_{\boldsymbol{\phi} \boldsymbol{x}}^{\phi}\right), A_{T, K}$ is a Poisson random variable with parameter $\int_{T}^{\infty} \int_{\langle\mathbf{1}, \boldsymbol{z}\rangle>K \mathrm{e}^{\lambda_{1} s}} z\left(\eta_{s}\right) \mu_{\eta_{s}}(\mathrm{~d} \boldsymbol{z}) \mathrm{d} s$. To prove (2.6), we only need to prove that

$$
\int_{T}^{\infty} \mathrm{d} s \int_{\langle\mathbf{1}, \boldsymbol{z}\rangle>K \mathrm{e}^{\lambda_{1} s}} z\left(\eta_{s}\right) \mu_{\eta_{s}}(\mathrm{~d} \boldsymbol{z})=\infty, \quad \boldsymbol{P}_{\boldsymbol{\phi} \boldsymbol{x}}^{\phi} \text { a.s. }
$$

for all $T, K>1$. Observe that $\eta$ is ergodic. Then there exists $C_{i}>0$ and random $S$ such that

$$
\int_{0}^{t} \mathbf{1}_{\left\{\eta_{s}=i\right\}} \mathrm{d} s \geq C_{i} t \quad \text { for all } t \geq S \quad \boldsymbol{P}_{\phi \boldsymbol{x}}^{\phi} \text { a.s. }
$$


Let us denote by $R=\max \left\{K \mathrm{e}^{\lambda_{1} T}, K \mathrm{e}^{\lambda_{1} S}\right\}$. Then, by Fubini's Theorem and the previous inequality,

$$
\begin{aligned}
\int_{T}^{\infty} \int_{\langle\mathbf{1}, \boldsymbol{z}\rangle>K \mathrm{e}^{\lambda_{1} s}} z\left(\eta_{s}\right) \mu_{\eta_{s}}(\mathrm{~d} \boldsymbol{z}) \mathrm{d} s & \geq \int_{T}^{\infty} \int_{\langle\mathbf{1}, \boldsymbol{z}\rangle>K \mathrm{e}^{\lambda_{1} s}} z\left(\eta_{s}\right) \mathbf{1}_{\left\{\eta_{s}=i\right\}} \mu_{\eta_{s}}(\mathrm{~d} \boldsymbol{z}) \mathrm{d} s \\
& \geq \frac{C_{i}}{\lambda_{1}} \int_{\langle\mathbf{1}, \boldsymbol{z}\rangle>R} z(i) \ln (\langle\mathbf{1}, \boldsymbol{z}\rangle) \mu_{i}(\mathrm{~d} \boldsymbol{z})-D=\infty,
\end{aligned}
$$

where $D=\left(\lambda_{1}^{-1} \ln (K)+T\right) \int_{\langle\mathbf{1}, \boldsymbol{z}\rangle>R} z(i) \mu_{i}(\mathrm{~d} \boldsymbol{z})<\infty$. Therefore we have (2.5).

(ii) Next suppose that, for $j \neq i$,

$$
\int_{\langle\mathbf{1}, \boldsymbol{z}\rangle>1} z(j) \ln (\langle\mathbf{1}, \boldsymbol{z}\rangle) \mu_{i}(\mathrm{~d} \boldsymbol{z})=\infty .
$$

By inequality (1.5) and the ergodicity of $\eta$, the set $\tau:=\left\{s \geq 0: \eta_{s-}=i, \eta_{s}=j\right\}$ is not bounded. For all $s \in \mathcal{T}$

$$
Z_{s} \geq \mathrm{e}^{-\lambda_{1} s}\left\langle\boldsymbol{\phi}, \boldsymbol{X}_{0}^{j, s}\right\rangle
$$

Let us denote by $\tau_{1}, \tau_{2}, \cdots$ a enumeration of this times in increasing order. By applying the distribution of $\boldsymbol{X}^{j, \cdot}$ in the jump times $\tau_{n}$ we have that for all $K>0$,

$$
\begin{aligned}
\sum_{n \geq 1} \mathrm{P}_{\boldsymbol{x}}\left(\mathrm{e}^{-\lambda_{1} \tau_{n}}\left\langle\mathbf{1}, \boldsymbol{X}_{0}^{j, \tau_{n}}\right\rangle \geq K\right) & =\sum_{n \geq 1} \mathrm{E}_{\boldsymbol{x}}\left[\mathrm{P}_{\boldsymbol{x}}\left(\mathrm{e}^{-\lambda_{1} \tau_{n}}\left\langle\mathbf{1}, \boldsymbol{X}_{0}^{j, \tau_{n}}\right\rangle \geq K \mid \tau_{n}\right)\right] \\
& =\frac{1}{B_{i j}^{T}} \sum_{n \geq 1} \mathrm{E}_{\boldsymbol{x}}\left[\int_{\mathbb{R}_{+}^{d}} \mathbf{1}_{\left\{\mathrm{e}^{-\lambda_{1} \tau_{n}}\langle\mathbf{1}, \boldsymbol{z}\rangle \geq K\right\}} z(j) \mu_{i}(\mathrm{~d} \boldsymbol{z})\right] \\
& =\frac{1}{B_{i j}^{T}} \mathrm{E}_{\boldsymbol{x}}\left[\sum_{n \geq 1} \int_{\mathbb{R}_{+}^{d}} \mathbf{1}_{\left\{\mathrm{e}^{-\lambda_{1} \tau_{n}}\langle\mathbf{1}, \boldsymbol{z}\rangle \geq K\right\}} z(j) \mu_{i}(\mathrm{~d} \boldsymbol{z})\right]
\end{aligned}
$$

By renewal theory, there exist $A \in(0, \infty)$ and a subset $\Omega_{1}$ with $\mathrm{P}_{\boldsymbol{x}}\left(\Omega_{1}\right)=1$ such that for all $\omega \in \Omega_{1}$ there exists $N=N(\omega)>0$ such that

$$
\tau_{n} \leq A n, \quad \text { for all } n \geq N \text {. }
$$

Then,

$$
\sum_{n \geq 1} \int_{\mathbb{R}_{+}^{d}} \mathbf{1}_{\left\{\mathrm{e}^{-\lambda_{1} \tau_{n}}\langle\mathbf{1}, \boldsymbol{z}\rangle \geq K\right\}} z(j) \mu_{i}(\mathrm{~d} \boldsymbol{z}) \geq \sum_{n \geq N} \int_{\mathbb{R}_{+}^{d}} \mathbf{1}_{\left\{\mathrm{e}^{-\lambda_{1} A n}\langle\mathbf{1}, \boldsymbol{z}\rangle \geq K\right\}} z(j) \mu_{i}(\mathrm{~d} \boldsymbol{z}) .
$$

By the integral test criterion for series, the previous series is divergent since

$$
\begin{aligned}
& \int_{N}^{\infty} \int_{\mathbb{R}_{+}^{d}} \mathbf{1}_{\left\{\mathrm{e}^{-\lambda_{1} A s}\langle\mathbf{1}, \boldsymbol{z}\rangle \geq K\right\}} z(j) \mu_{i}(\mathrm{~d} \boldsymbol{z}) \mathrm{d} s \\
& =\frac{1}{A \lambda_{1}} \int_{\langle\mathbf{1}, \boldsymbol{z}\rangle>K} z(j) \ln (\langle\mathbf{1}, \boldsymbol{z}\rangle) \mu_{i}(\mathrm{~d} \boldsymbol{z})-D=\infty,
\end{aligned}
$$

where $D=\left(\left(\lambda_{1} A\right)^{-1} \ln (K)+N\right) \int_{\langle\mathbf{1}, \boldsymbol{z}\rangle>K} \boldsymbol{z}(i) \mu_{i}(\mathrm{~d} \boldsymbol{z})<\infty$. This implies

$$
\begin{aligned}
& \sum_{n \geq 1} \mathrm{P}_{\boldsymbol{x}}\left(\mathrm{e}^{-\lambda_{1} \tau_{n}}\left\langle\mathbf{1}, \boldsymbol{X}_{0}^{j, \tau_{n}}\right\rangle \geq K\right) \\
& =\mathrm{E}_{\boldsymbol{x}}\left[\sum_{n \geq 1} \int_{\mathbb{R}_{+}^{d}} \mathbf{1}_{\left\{\mathrm{e}^{-\lambda_{1} \tau_{n}}\langle\mathbf{1}, \boldsymbol{z}\rangle \geq K\right\}} z(j) \mu_{i}(\mathrm{~d} \boldsymbol{z})\right]=\infty .
\end{aligned}
$$


Therefore, by the Borel Cantelli Lemma,

$$
\limsup _{n \rightarrow \infty} \mathrm{e}^{-\lambda_{1} \tau_{n}}\left\langle\mathbf{1}, \boldsymbol{X}_{0}^{j, \tau_{n}}\right\rangle \geq K, \quad \mathrm{P}_{\boldsymbol{x}} \text { a.s. }
$$

for any $K>0$, and then

$$
\limsup _{n \rightarrow \infty} \mathrm{e}^{-\lambda_{1} \tau_{n}}\left\langle\mathbf{1}, \boldsymbol{X}_{0}^{j, \tau_{n}}\right\rangle=\infty
$$

Hence we have

$$
\limsup _{s \rightarrow \infty} Z_{s} \geq \limsup _{n \rightarrow \infty} \mathrm{e}^{-\lambda_{1} \tau_{n}}\left\langle\boldsymbol{\phi}, \boldsymbol{X}_{0}^{j, \tau_{n}}\right\rangle=\infty,
$$

which says that (2.5) holds.

\section{Proof of Theorem 1.4}

Suppose that ( $x \log x$ condition) doesn't hold, then

$$
\limsup _{t \rightarrow \infty} \mathrm{e}^{-\lambda_{1} t} \boldsymbol{X}_{t}(i) \leq \lim _{t \rightarrow \infty} \frac{1}{\phi(i)} \mathrm{e}^{-\lambda_{1} t}\left\langle\boldsymbol{\phi}, \boldsymbol{X}_{t}\right\rangle=0 \quad \mathbb{P}_{\boldsymbol{x}} \text { a.s. }
$$

We therefore focus on the case when $(x \log x$ condition) holds. In order to do this, we will separate small jumps from big jumps in the Poisson measures. More precisely, for each $i \in E$, let us define the Poisson random measures

$$
N_{i}^{(1)}(\mathrm{d} s, \mathrm{~d} \boldsymbol{z}, \mathrm{d} r):=\mathbf{1}_{\left\{\langle\mathbf{1}, \boldsymbol{z}\rangle \leq \mathrm{e}^{\lambda_{1} s}\right\}} N_{i}(\mathrm{~d} s, \mathrm{~d} \boldsymbol{z}, \mathrm{d} r)
$$

and

$$
N_{i}^{(2)}(\mathrm{d} s, \mathrm{~d} \boldsymbol{z}, \mathrm{d} r):=\mathbf{1}_{\left\{\langle\mathbf{1}, \boldsymbol{z}\rangle>\mathrm{e}^{\lambda_{1} s}\right\}} N_{i}(\mathrm{~d} s, \mathrm{~d} \boldsymbol{z}, \mathrm{d} r)
$$

and denote by $\widetilde{N}_{i}^{(1)}$ and $\widetilde{N}_{i}^{(2)}$ their compensated versions, respectively.

We are going to compute the proof of Theorem 1.4 in three steps. First, in lattice times, we will approximate the value of the limit by the value of the limit of a conditional expectation. With this relation, we are going to find our limit in lattice times. And finally in the third step, we will extend the result to continuous times.

3.1. Proof for lattice times. First, we will prove Theorem 1.4 in lattice times. For each $\delta>0$, consider the lattice times $n \delta, n \in \mathbb{N}$. We will approximate the value of the limit by the value of the limit of a conditional expectation.

Lemma 3.1. If ( $x \log x$ condition) holds, then for any $m \in \mathbb{N}, \sigma>0$ and $\boldsymbol{x} \in \mathbb{R}_{+}^{d}$,

$$
\lim _{n \rightarrow \infty} \mathrm{e}^{-\lambda_{1}(n+m) \sigma} \boldsymbol{X}_{(n+m) \sigma}-\mathbb{E}_{\boldsymbol{x}}\left[\mathrm{e}^{-\lambda_{1}(n+m) \sigma} \boldsymbol{X}_{(n+m) \sigma} \mid \mathcal{F}_{n \sigma}\right]=0,
$$

in $\mathbb{L}^{1}\left(\mathbb{P}_{\boldsymbol{x}}\right)$ and $\mathbb{P}_{\boldsymbol{x}}$ a.s.

Proof: The result is true if we prove that for all $k \in E$,

$$
\lim _{n \rightarrow \infty} \mathrm{e}^{-\lambda_{1}(n+m) \sigma}\left\langle\boldsymbol{e}_{k}, \boldsymbol{X}_{(n+m) \sigma}\right\rangle-\mathbb{E}_{\boldsymbol{x}}\left[\mathrm{e}^{-\lambda_{1}(n+m) \sigma}\left\langle\boldsymbol{e}_{k}, \boldsymbol{X}_{(n+m) \sigma}\right\rangle \mid \mathcal{F}_{n \sigma}\right]=0,
$$

in $\mathbb{L}^{1}\left(\mathbb{P}_{\boldsymbol{x}}\right)$ and $\mathbb{P}_{\boldsymbol{x}}$ a.s. Let $s, t \geq 0$. By the Markov property we have

$$
\begin{gathered}
\mathrm{e}^{-\lambda_{1}(t+s)}\left\langle\boldsymbol{e}_{k}, \boldsymbol{X}_{t+s}\right\rangle-\mathbb{E}_{\boldsymbol{x}}\left[\mathrm{e}^{-\lambda_{1}(t+s)}\left\langle\boldsymbol{e}_{k}, \boldsymbol{X}_{t+s}\right\rangle \mid \mathcal{F}_{t}\right] \\
=\mathrm{e}^{-\lambda_{1}(t+s)}\left\langle\boldsymbol{e}_{k}, \boldsymbol{X}_{t+s}\right\rangle-\mathrm{e}^{-\lambda_{1}(t+s)}\left\langle\boldsymbol{M}(s) \boldsymbol{e}_{k}, \boldsymbol{X}_{t}\right\rangle .
\end{gathered}
$$


Now, applying equation (1.8) to the times $t$ and $t+s$, we obtain

$$
\begin{aligned}
& \mathrm{e}^{-\lambda_{1}(t+s)}\left\langle\boldsymbol{e}_{k}, \boldsymbol{X}_{t+s}\right\rangle-\mathrm{e}^{-\lambda_{1}(t+s)}\left\langle\boldsymbol{M}(s) \boldsymbol{e}_{k}, \boldsymbol{X}_{t}\right\rangle \\
= & \sum_{i \in E} \mathrm{e}^{-\lambda_{1}(t+s)} \int_{t}^{t+s}\left[\boldsymbol{M}(t+s-u) \boldsymbol{e}_{k}\right]_{i} \sqrt{2 c_{i} X_{u, i}} \mathrm{~d} W_{u, i} \\
& +\sum_{i \in E} \mathrm{e}^{-\lambda_{1}(t+s)} \int_{t}^{t+s} \int_{\mathbb{R}_{+}^{d}} \int_{0}^{\infty}\left\langle\boldsymbol{M}(t+s-u) \boldsymbol{e}_{k}, \boldsymbol{z}\right\rangle \mathbf{1}_{\left\{l \leq X_{u-, i}\right\}} \widetilde{N}_{i}(\mathrm{~d} u, \mathrm{~d} \boldsymbol{z}, \mathrm{d} l) \\
= & C_{t, t+s}\left(\boldsymbol{e}_{k}\right)+S_{t, t+s}\left(\boldsymbol{e}_{k}\right)+B_{t, t+s}\left(\boldsymbol{e}_{k}\right),
\end{aligned}
$$

where

$$
\begin{aligned}
C_{t, t+s}\left(\boldsymbol{e}_{k}\right) & :=\sum_{i \in E} \mathrm{e}^{-\lambda_{1}(t+s)} \int_{t}^{t+s}\left[\boldsymbol{M}(t+s-u) \boldsymbol{e}_{k}\right] i \sqrt{2 c_{i} X_{u, i}} \mathrm{~d} W_{u, i}, \\
S_{t, t+s}\left(\boldsymbol{e}_{k}\right) & :=\sum_{i \in E} \mathrm{e}^{-\lambda_{1}(t+s)} \int_{t}^{t+s} \int_{\mathbb{R}_{+}^{d}} \int_{0}^{\infty}\left\langle\boldsymbol{M}(t+s-u) \boldsymbol{e}_{k}, \boldsymbol{z}\right\rangle \mathbf{1}_{\left\{r \leq X_{u-, i}\right\}} \widetilde{N}_{i}^{(1)}(\mathrm{d} u, \mathrm{~d} \boldsymbol{z}, \mathrm{d} r), \\
B_{t, t+s}\left(\boldsymbol{e}_{k}\right): & =\sum_{i \in E} \mathrm{e}^{-\lambda_{1}(t+s)} \int_{t}^{t+s} \int_{\mathbb{R}_{+}^{d}} \int_{0}^{\infty}\left\langle\boldsymbol{M}(t+s-u) \boldsymbol{e}_{k}, \boldsymbol{z}\right\rangle \mathbf{1}_{\left\{r \leq X_{u-, i}\right\}} \widetilde{N}_{i}^{(2)}(\mathrm{d} u, \mathrm{~d} \boldsymbol{z}, \mathrm{d} r) .
\end{aligned}
$$

To complete the proof, we need to control the convergence of the above three terms.

(i) Lattice convergence of $C_{t, t+s}\left(\boldsymbol{e}_{k}\right)$ : We will show that for any $k \in E, m \in \mathbb{N}$, $\sigma>0$ and $\boldsymbol{x} \in \mathbb{R}_{+}^{d}$

$$
\lim _{n \rightarrow \infty} C_{n \sigma,(n+m) \sigma}\left(\boldsymbol{e}_{k}\right)=0, \quad \text { in } \mathbb{L}^{2}\left(\mathbb{P}_{\boldsymbol{x}}\right) \text { and } \mathbb{P}_{\boldsymbol{x}} \text { a.s. }
$$

First note that for $t \in[n \sigma,(n+m) \sigma]$, the process

$$
C_{t}^{(n, m, \sigma)}:=\sum_{i \in E} \mathrm{e}^{-\lambda_{1}(n+m) \sigma} \int_{n \sigma}^{t}\left[\boldsymbol{M}((n+m) \sigma-u) \boldsymbol{e}_{k}\right]_{i} \sqrt{2 c_{i} X_{u, i}} \mathrm{~d} W_{u, i}
$$

is a continuous local martingale with quadratic variation given by

$$
\sum_{i \in E} \mathrm{e}^{-2 \lambda_{1}(n+m) \sigma} \int_{n \sigma}^{t}\left(\left[\boldsymbol{M}((n+m) \sigma-u) \boldsymbol{e}_{k}\right]_{i}\right)^{2} 2 c_{i} X_{u, i} \mathrm{~d} u
$$

Then, by taking $t=(n+m) \sigma$, we have

$$
\begin{aligned}
& \mathbb{E}_{\boldsymbol{x}}\left[\left(C_{n \sigma,(n+m) \sigma}\left(\boldsymbol{e}_{k}\right)\right)^{2}\right] \\
& =\mathrm{e}^{-2 \lambda_{1}(n+m) \sigma} \mathbb{E}_{\boldsymbol{x}}\left[\sum_{i \in E} \int_{n \sigma}^{(n+m) \sigma}\left(\left[\boldsymbol{M}((n+m) \sigma-u) \boldsymbol{e}_{k}\right]_{i}\right)^{2} 2 c_{i} X_{u, i} \mathrm{~d} u\right] .
\end{aligned}
$$

Denote by $C=\max \left\{c_{i}: i \in E\right\}$. Observe that $\left(\boldsymbol{M}(t) \boldsymbol{e}_{k}\right)_{i}=M(t)_{i, k}$ and by equation (1.9) exists $C_{1}>0$ such that $\|\boldsymbol{M}(t)\| \leq C_{1} \mathrm{e}^{\lambda_{1} t}$. Recall that $m_{\phi}=$ 
$\min \{\phi(i): i \in E\}$. Then

$$
\begin{aligned}
& \mathbb{E}_{\boldsymbol{x}}\left[\left(C_{n \sigma,(n+m) \sigma}\left(\boldsymbol{e}_{k}\right)\right)^{2}\right] \\
& \leq \frac{2 C C_{1}^{2}}{m_{\boldsymbol{\phi}}} \mathrm{e}^{-2 \lambda_{1}(n+m) \sigma} \mathbb{E}_{\boldsymbol{x}}\left[\int_{n \sigma}^{(n+m) \sigma} \mathrm{e}^{2 \lambda_{1}[(n+m) \sigma-u]}\left\langle\boldsymbol{\phi}, \boldsymbol{X}_{u}\right\rangle \mathrm{d} u\right] \\
& =\frac{2 C C_{1}^{2}\langle\boldsymbol{\phi}, \boldsymbol{x}\rangle}{\lambda_{1} m_{\boldsymbol{\phi}}}\left[\mathrm{e}^{-\lambda_{1} n \sigma}-\mathrm{e}^{-\lambda_{1}(n+m) \sigma}\right] \\
& =\frac{2 C C_{1}^{2}\langle\boldsymbol{\phi}, \boldsymbol{x}\rangle}{\lambda_{1} m_{\boldsymbol{\phi}}}\left[1-\mathrm{e}^{-\lambda_{1} m \sigma}\right] \mathrm{e}^{-\lambda_{1} n \sigma},
\end{aligned}
$$

where in the first equality we used the fact that $W_{u}=\mathrm{e}^{-\lambda_{1} u}\left\langle\boldsymbol{\phi}, \boldsymbol{X}_{u}\right\rangle$ is a martingale. Therefore

$$
\sum_{n=1}^{\infty} \mathbb{E}_{\boldsymbol{x}}\left[\left(C_{n \sigma,(n+m) \sigma}\left(\boldsymbol{e}_{k}\right)\right)^{2}\right]<\infty .
$$

Then we have the $\mathbb{L}^{2}\left(\mathbb{P}_{\boldsymbol{x}}\right)$-convergence. The $\mathbb{P}_{\boldsymbol{x}}$ a.s. convergence follows from Chebyshev's inequality, Borel-Cantelli Lemma and the previous inequality.

(ii) Lattice convergence of $S_{t, t+s}\left(\boldsymbol{e}_{k}\right)$ : We will show that, if ( $x \log x$ condition) holds, then for any $k \in E, m \in \mathbb{N}, \sigma>0$ and $\boldsymbol{x} \in \mathbb{R}_{+}^{d}$,

$$
\lim _{n \rightarrow \infty} S_{n \sigma,(n+m) \sigma}\left(\boldsymbol{e}_{k}\right)=0, \quad \text { in } \mathbb{L}^{2}\left(\mathbb{P}_{\boldsymbol{x}}\right) \text { and } \mathbb{P}_{\boldsymbol{x}} \text { a.s. }
$$

Similar to the proof in (i) above, for $t \in[n \sigma,(n+m) \sigma]$, the process,

$$
\begin{aligned}
& S_{t}^{(n, m, \sigma)} \\
& :=\mathrm{e}^{-\lambda_{1}(n+m) \sigma} \sum_{i \in E} \int_{n \sigma}^{t} \int_{\mathbb{R}_{+}^{d}} \int_{0}^{\infty}\left\langle\boldsymbol{M}((n+m) \sigma-u) \boldsymbol{e}_{k}, \boldsymbol{z}\right\rangle \mathbf{1}_{\left\{r \leq X_{u-, i}\right\}} \widetilde{N}_{i}^{(1)}(\mathrm{d} u, \mathrm{~d} \boldsymbol{z}, \mathrm{d} r)
\end{aligned}
$$

is a martingale with quadratic variation given by

$$
\begin{array}{r}
\mathrm{e}^{-2 \lambda_{1}(n+m) \sigma} \sum_{i \in E} \int_{n \sigma}^{t} \int_{\mathbb{R}_{+}^{d}} \int_{0}^{\infty}\left\langle\boldsymbol{M}((n+m) \sigma-u) \boldsymbol{e}_{k}, \boldsymbol{z}\right\rangle^{2} \\
\mathbf{1}_{\left\{r \leq X_{u, i}\right\}} \mathbf{1}_{\left\{\langle\mathbf{1}, \boldsymbol{z}\rangle \leq \mathrm{e}^{\lambda_{1} u}\right\}} \mathrm{d} r \mu_{i}(\mathrm{~d} \boldsymbol{z}) \mathrm{d} u .
\end{array}
$$

Then, by taking $t=(n+m) \sigma$, we have

$$
\begin{aligned}
\mathbb{E}_{\boldsymbol{x}}\left[\left(S_{n \sigma,(n+m) \sigma}\left(\boldsymbol{e}_{k}\right)\right)^{2}\right] & \mathrm{e}^{-2 \lambda_{1}(n+m) \sigma} \sum_{\mathbb{E}_{\boldsymbol{x}}}\left[\int_{n \sigma}^{(n+m) \sigma} \int_{\mathbb{R}_{+}^{d}}\left\langle\boldsymbol{M}((n+m) \sigma-u) \boldsymbol{e}_{k}, \boldsymbol{z}\right\rangle^{2}\right. \\
& \left.X_{u, i} \mathbf{1}_{\left\{\langle\mathbf{1}, \boldsymbol{z}\rangle \leq \mathrm{e}^{\lambda_{1} u}\right\}} \mu_{i}(\mathrm{~d} \boldsymbol{z}) \mathrm{d} u\right] .
\end{aligned}
$$

By equation (1.9), there exists $C>0$ such that for all $t \geq 0$,

$$
\left\langle\boldsymbol{M}(t) \boldsymbol{e}_{k}, \boldsymbol{z}\right\rangle \leq C \mathrm{e}^{\lambda_{1} t}\langle\mathbf{1}, \boldsymbol{z}\rangle \text {. }
$$


Therefore, by using the definition of $m_{\phi}$

$$
\begin{aligned}
& \mathbb{E}_{\boldsymbol{x}}\left[\left(S_{n \sigma,(n+m) \sigma}\left(\boldsymbol{e}_{k}\right)\right)^{2}\right] \\
& \leq \frac{C^{2}}{m_{\boldsymbol{\phi}}} \sum_{i \in E} \mathbb{E}_{\boldsymbol{x}}\left[\int_{n \sigma}^{(n+m) \sigma} \int_{\mathbb{R}_{+}^{d}} \mathrm{e}^{-2 \lambda_{1} u}\langle\mathbf{1}, \boldsymbol{z}\rangle^{2}\left\langle\boldsymbol{\phi}, \boldsymbol{X}_{u}\right\rangle \mathbf{1}_{\left\{\langle\mathbf{1}, \boldsymbol{z}\rangle \leq \mathrm{e}^{\lambda_{1} u}\right\}} \mu_{i}(\mathrm{~d} \boldsymbol{z}) \mathrm{d} u\right] \\
& =\frac{C^{2}\langle\boldsymbol{\phi}, \boldsymbol{x}\rangle}{m_{\boldsymbol{\phi}}} \sum_{i \in E} \int_{n \sigma}^{(n+m) \sigma} \int_{\mathbb{R}_{+}^{d}} \mathrm{e}^{-\lambda_{1} u}\langle\mathbf{1}, \boldsymbol{z}\rangle^{2} \mathbf{1}_{\left\{\langle\mathbf{1}, \boldsymbol{z}\rangle \leq \mathrm{e}^{\lambda_{1} u}\right\}} \mu_{i}(\mathrm{~d} \boldsymbol{z}) \mathrm{d} u,
\end{aligned}
$$

where in the first equality we used the fact that $W_{u}=\mathrm{e}^{-\lambda_{1} u}\left\langle\boldsymbol{\phi}, \boldsymbol{X}_{u}\right\rangle$ is a martingale. Taking sum over $n$, we get

$$
\begin{aligned}
& \sum_{n=1}^{\infty} \mathbb{E}_{\boldsymbol{x}}\left[\left(S_{n \sigma,(n+m) \sigma}\left(\boldsymbol{e}_{k}\right)\right)^{2}\right] \\
& \leq \frac{C^{2}\langle\boldsymbol{\phi}, \boldsymbol{x}\rangle}{m_{\boldsymbol{\phi}}} \sum_{n=1}^{\infty} \sum_{i \in E} \int_{n \sigma}^{\infty} \int_{\mathbb{R}_{+}^{d}} \mathrm{e}^{-\lambda_{1} u}\langle\mathbf{1}, \boldsymbol{z}\rangle^{2} \mathbf{1}_{\left\{\langle\mathbf{1}, \boldsymbol{z}\rangle \leq \mathrm{e}^{\lambda_{1} u}\right\}} \mu_{i}(\mathrm{~d} \boldsymbol{z}) \mathrm{d} u .
\end{aligned}
$$

By Fubini's Theorem applied to the Lebesgue measure in $\mathbb{R}$ and the countable measure in $\mathbb{N}$, we get

$$
\begin{aligned}
& \sum_{n=1}^{\infty} \mathbb{E}_{\boldsymbol{x}}\left[\left(S_{n \sigma,(n+m) \sigma}\left(\boldsymbol{e}_{k}\right)\right)^{2}\right] \\
& \leq \frac{C^{2}\langle\boldsymbol{\phi}, \boldsymbol{x}\rangle}{m_{\boldsymbol{\phi}}} \sum_{i \in E} \int_{\sigma}^{\infty} \sum_{n=1}^{\lfloor u / \sigma\rfloor} \int_{\mathbb{R}_{+}^{d}} \mathrm{e}^{-\lambda_{1} u}\langle\mathbf{1}, \boldsymbol{z}\rangle^{2} \mathbf{1}_{\left\{\langle\mathbf{1}, \boldsymbol{z}\rangle \leq \mathrm{e}^{\lambda_{1} u}\right\}} \mu_{i}(\mathrm{~d} \boldsymbol{z}) \mathrm{d} u \\
& \leq \frac{C^{2}\langle\boldsymbol{\phi}, \boldsymbol{x}\rangle}{\sigma m_{\boldsymbol{\phi}}} \sum_{i \in E} \int_{0}^{\infty} \int_{\mathbb{R}_{+}^{d}} u \mathrm{e}^{-\lambda_{1} u}\langle\mathbf{1}, \boldsymbol{z}\rangle^{2} \mathbf{1}_{\left\{\langle\mathbf{1}, \boldsymbol{z}\rangle \leq \mathrm{e}^{\lambda_{1} u}\right\}} \mu_{i}(\mathrm{~d} \boldsymbol{z}) \mathrm{d} u .
\end{aligned}
$$

By Fubini's Theorem, for each $i \in E$,

$$
\begin{aligned}
& \int_{0}^{\infty} \int_{\mathbb{R}_{+}^{d}} u \mathrm{e}^{-\lambda_{1} u}\langle\mathbf{1}, \boldsymbol{z}\rangle^{2} \mathbf{1}_{\left\{\langle\mathbf{1}, \boldsymbol{z}\rangle \leq \mathrm{e}^{\lambda_{1} u}\right\}} \mu_{i}(\mathrm{~d} \boldsymbol{z}) \mathrm{d} u \\
& =\int_{\langle\mathbf{1}, \boldsymbol{z}\rangle \leq 1} \int_{0}^{\infty} u \mathrm{e}^{-\lambda_{1} u}\langle\mathbf{1}, \boldsymbol{z}\rangle^{2} \mu_{i}(\mathrm{~d} \boldsymbol{z}) \mathrm{d} u \\
& +\int_{\langle\mathbf{1}, \boldsymbol{z}\rangle>1} \int_{\lambda_{1}^{-1} \ln (\langle\mathbf{1}, \boldsymbol{z}\rangle)}^{\infty} u \mathrm{e}^{-\lambda_{1} u}\langle\mathbf{1}, \boldsymbol{z}\rangle^{2} \mathrm{~d} u \mu_{i}(\mathrm{~d} \boldsymbol{z}) \\
& =\frac{1}{\lambda_{1}^{2}}\left(\int_{\langle\mathbf{1}, \boldsymbol{z}\rangle \leq 1}\langle\mathbf{1}, \boldsymbol{z}\rangle^{2} \mu_{i}(\mathrm{~d} \boldsymbol{z})+\int_{\langle\mathbf{1}, \boldsymbol{z}\rangle>1}\langle\mathbf{1}, \boldsymbol{z}\rangle(\ln (\langle\mathbf{1}, \boldsymbol{z}\rangle)+1) \mu_{i}(\mathrm{~d} \boldsymbol{z})\right)<\infty .
\end{aligned}
$$

Since ( $x \log x$ condition) holds,

$$
\sum_{n=1}^{\infty} \mathbb{E}_{\boldsymbol{x}}\left[\left(S_{n \sigma,(n+m) \sigma}\left(\boldsymbol{e}_{k}\right)\right)^{2}\right]<\infty
$$

and we have the convergence in $\mathbb{L}^{2}\left(\mathbb{P}_{\boldsymbol{x}}\right)$. By Chebyshev's inequality and BorelCantelli Lemma we have the $\mathbb{P}_{\boldsymbol{x}}$ a.s. convergence. 
(iii) Lattice convergence of $B_{t, t+s}\left(\boldsymbol{e}_{k}\right)$ : We show that if ( $x \log x$ condition) holds, then for any $k \in E, m \in \mathbb{N}, \sigma>0$ and $\boldsymbol{x} \in \mathbb{R}_{+}^{d}$,

$$
\lim _{n \rightarrow \infty} B_{n \sigma,(n+m) \sigma}\left(\boldsymbol{e}_{k}\right)=0, \quad \text { in } \mathbb{L}^{1}\left(\mathbb{P}_{\boldsymbol{x}}\right) \text { and } \mathbb{P}_{\boldsymbol{x}} \text { a.s. }
$$

Note that for any random measure $N$, we have $\widetilde{N}(A) \leq N(A)+\widehat{N}(A)$, where $\widetilde{N}$ is the compensated measure and $\widehat{N}$ the intensity measure. Then, by inequality $(3.5)$

$$
\begin{aligned}
& \left|B_{n \sigma,(n+m) \sigma}\left(\boldsymbol{e}_{k}\right)\right| \\
& \leq C \sum_{i \in E} \int_{n \sigma}^{(n+m) \sigma} \int_{\mathbb{R}_{+}^{d}} \int_{0}^{\infty} \mathrm{e}^{-\lambda u}\langle\mathbf{1}, \boldsymbol{z}\rangle \mathbf{1}_{\left\{r \leq X_{u, i}\right\}} \mathbf{1}_{\left\{\langle\mathbf{1}, \boldsymbol{z}\rangle>\mathrm{e}^{\lambda_{1} u}\right\}}\left(\mathrm{d} N_{i}+\mathrm{d} r \mu_{i}(\mathrm{~d} \boldsymbol{z}) \mathrm{d} u\right) \\
& \leq C \sum_{i \in E} \int_{n \sigma}^{\infty} \int_{\mathbb{R}_{+}^{d}} \int_{0}^{\infty} \mathrm{e}^{-\lambda u}\langle\mathbf{1}, \boldsymbol{z}\rangle \mathbf{1}_{\left\{r \leq X_{u, i}\right\}} \mathbf{1}_{\left\{\langle\mathbf{1}, \boldsymbol{z}\rangle>\mathrm{e}^{\lambda_{1} u}\right\}}\left(\mathrm{d} N_{i}+\mathrm{d} r \mu_{i}(\mathrm{~d} \boldsymbol{z}) \mathrm{d} u\right) .
\end{aligned}
$$

Using the fact that $W$ is a martingale, we have

$$
\begin{aligned}
& \mathbb{E}_{\boldsymbol{x}}\left[\left|B_{n \sigma,(n+m) \sigma}\left(\boldsymbol{e}_{k}\right)\right|\right] \\
& \leq \frac{2 C}{m_{\boldsymbol{\phi}}} \sum_{i \in E} \mathbb{E}_{\boldsymbol{x}}\left[\int_{n \sigma}^{\infty} \int_{\mathbb{R}_{+}^{d}} \mathrm{e}^{-\lambda u}\langle\mathbf{1}, \boldsymbol{z}\rangle\left\langle\boldsymbol{\phi}, X_{u}\right\rangle \mathbf{1}_{\left\{\langle\mathbf{1}, \boldsymbol{z}\rangle>\mathrm{e}^{\lambda_{1} u}\right\}} \mu_{i}(\mathrm{~d} \boldsymbol{z}) \mathrm{d} u\right] . \\
& =\frac{2 C\langle\boldsymbol{\phi}, \boldsymbol{x}\rangle}{m_{\boldsymbol{\phi}}} \sum_{i \in E} \int_{n \sigma}^{\infty} \int_{\mathbb{R}_{+}^{d}}\langle\mathbf{1}, \boldsymbol{z}\rangle \mathbf{1}_{\left\{\langle\mathbf{1}, \boldsymbol{z}\rangle>\mathrm{e}^{\lambda_{1} u}\right\}} \mu_{i}(\mathrm{~d} \boldsymbol{z}) \mathrm{d} u .
\end{aligned}
$$

By Fubini's Theorem,

$$
\mathbb{E}_{\boldsymbol{x}}\left[\left|B_{n \sigma,(n+m) \sigma}\left(\boldsymbol{e}_{k}\right)\right|\right] \leq \frac{2 C\langle\boldsymbol{\phi}, \boldsymbol{x}\rangle}{m_{\boldsymbol{\phi}} \lambda_{1}} \sum_{i \in E} \int_{\langle\mathbf{1}, \boldsymbol{z}\rangle>\mathrm{e}^{\lambda_{1} \sigma n}}\langle\mathbf{1}, \boldsymbol{z}\rangle \ln (\langle\mathbf{1}, \boldsymbol{z}\rangle) \mu_{i}(\mathrm{~d} \boldsymbol{z}) .
$$

Recall that $\int_{1 \leq\langle\mathbf{1}, \boldsymbol{z}\rangle<\infty}\langle\mathbf{1}, \boldsymbol{z}\rangle \ln (\langle\mathbf{1}, \boldsymbol{z}\rangle) \mu_{i}(\mathrm{~d} \boldsymbol{z})<\infty$. Therefore

$$
\lim _{n \rightarrow \infty} \mathbb{E}_{\boldsymbol{x}}\left[\left|B_{n \sigma,(n+m) \sigma}\left(\boldsymbol{e}_{k}\right)\right|\right]=0,
$$

which says $\lim _{n \rightarrow \infty} B_{n \sigma,(n+m) \sigma}\left(\boldsymbol{e}_{k}\right)=0$ in $\mathbb{L}^{1}\left(\mathbb{P}_{\boldsymbol{x}}\right)$. The $\mathbb{P}_{\boldsymbol{x}}$ a.s. convergence follows from the fact that

$$
n \mapsto \int_{n \sigma}^{\infty} \int_{\mathbb{R}_{+}^{d}} \int_{0}^{\infty} \mathrm{e}^{-\lambda u}\langle\mathbf{1}, \boldsymbol{z}\rangle \mathbf{1}_{\left\{r \leq X_{u, i}\right\}} \mathbf{1}_{\left\{\langle\mathbf{1}, \boldsymbol{z}\rangle>\mathrm{e}^{\lambda_{1} u}\right\}}\left(\mathrm{d} N_{i}+\mathrm{d} r \mu_{i}(\mathrm{~d} \boldsymbol{z}) \mathrm{d} u\right)
$$

is decreasing and inequality (3.7).

Now applying (i)-(iii) to (3.1), the proof is complete.

Proposition 3.2. If ( $x \log x$ condition) holds, then for any $\sigma>0$,

$$
\lim _{n \rightarrow \infty} \mathrm{e}^{-\lambda_{1} n \sigma} \boldsymbol{X}_{n \sigma}=W_{\infty} \widehat{\boldsymbol{\phi}}, \quad \text { in } \mathbb{L}^{1}\left(\mathbb{P}_{\boldsymbol{x}}\right) \text { and } \mathbb{P}_{\boldsymbol{x}} \text { a.s. }
$$

Proof: Let $k \in E$ and $n, m>0$. By the Markov property, we have

$$
\mathbb{E}_{\boldsymbol{x}}\left[\mathrm{e}^{-\lambda_{1}(n+m) \sigma}\left\langle\boldsymbol{e}_{k}, \boldsymbol{X}_{(n+m) \sigma}\right\rangle \mid \mathcal{F}_{n \sigma}\right]=\mathrm{e}^{-\lambda_{1}(n+m) \sigma}\left\langle\boldsymbol{M}(m \sigma) \boldsymbol{e}_{k}, \boldsymbol{X}_{n \sigma}\right\rangle .
$$


Let

$$
r_{m}=\left(1-\frac{c_{1} \mathrm{e}^{-c_{2} m \sigma}}{\max \{\phi(i) \widehat{\phi}(k): i, k \in E\}}\right)
$$

and

$$
R_{m}=\left(1+\frac{c_{1} \mathrm{e}^{-c_{2} m \sigma}}{\min \{\phi(i) \widehat{\phi}(k): i, k \in E\}}\right) .
$$

Observe that $r_{m} \rightarrow 1$ and $R_{m} \rightarrow 1$ as $m \rightarrow \infty$. Moreover, by limit (1.9), for all $i \in E$

$$
r_{m} \phi(i) \widehat{\phi}(k) \leq \mathrm{e}^{-\lambda_{1} m \sigma}\left(\boldsymbol{M}(m \sigma) \boldsymbol{e}_{k}\right)_{i}=\mathrm{e}^{-\lambda_{1} m \sigma} M(m \sigma)_{i k} \leq R_{m} \phi(i) \widehat{\phi}(k) .
$$

Hence,

$$
\begin{aligned}
r_{m} \widehat{\phi}(k) \mathrm{e}^{-\lambda_{1} n \sigma}\left\langle\boldsymbol{\phi}, \boldsymbol{X}_{n \sigma}\right\rangle & \leq \mathrm{e}^{-\lambda_{1}(n+m) \sigma}\left\langle\boldsymbol{M}(m \sigma) \boldsymbol{e}_{k}, \boldsymbol{X}_{n \sigma}\right\rangle \\
& \leq R_{m} \widehat{\phi}(k) \mathrm{e}^{-\lambda_{1} n \sigma}\left\langle\boldsymbol{\phi}, \boldsymbol{X}_{n \sigma}\right\rangle
\end{aligned}
$$

Now, applying Lemma 3.1, the previous equation and equation (3.8), we get

$$
\begin{aligned}
\limsup _{n \rightarrow \infty} \mathrm{e}^{-\lambda_{1} n \sigma} X_{n \sigma}(k) & =\limsup _{m \rightarrow \infty} \limsup _{n \rightarrow \infty} \mathrm{e}^{-\lambda_{1}(n+m) \sigma}\left\langle\boldsymbol{e}_{k}, \boldsymbol{X}_{(n+m) \sigma}\right\rangle \\
& =\limsup _{m \rightarrow \infty} \limsup _{n \rightarrow \infty} \mathrm{e}^{-\lambda_{1}(n+m) \sigma}\left\langle\boldsymbol{M}(m \sigma) \boldsymbol{e}_{k}, \boldsymbol{X}_{n \sigma}\right\rangle \\
& \leq \lim _{m \rightarrow \infty} \lim _{n \rightarrow \infty} R_{m} \widehat{\phi}(k) W_{n \sigma} \\
& =\widehat{\phi}(k) W_{\infty}, \quad \mathbb{P}_{\boldsymbol{x}} \text { a.s. }
\end{aligned}
$$

In a similar way,

$$
\liminf _{n \rightarrow \infty} \mathrm{e}^{-\lambda_{1} n \sigma} X_{n \sigma}(k)=\widehat{\phi}(k) W_{\infty}, \quad \mathbb{P}_{\boldsymbol{x}} \text { a.s. }
$$

Therefore, the a.s. assertion is true. Recall that $m_{\phi}:=\min \{\phi(k), k \in E\}>0$. Observe that $0 \leq \mathrm{e}^{-\lambda_{1} n \sigma} X_{n \sigma}(k) \leq W_{n \sigma} m_{\phi}^{-1}$ and by Theorem 1.3 the martingale $W_{t}$ converges in $\mathbb{L}^{1}\left(\mathbb{P}_{\boldsymbol{x}}\right)$. Then by the Generalized Dominated Convergence Theorem, the $\mathbb{L}^{1}\left(\mathbb{P}_{\boldsymbol{x}}\right)$ assertion holds. (see for instance Dudley, 2002, Problem 12, p. 133),

3.2. From lattice times to continuous times. In this section, we extend the convergence along lattice times in Theorem 1.4 to convergence along continuous times and conclude our main results.

Proof of Theorem 1.4: Since $\boldsymbol{\phi}$ is a positive vector, then for any $\boldsymbol{a} \in \mathbb{R}^{d}$ we have $\left|\left\langle\boldsymbol{a}, \boldsymbol{X}_{t}\right\rangle\right| \leq \frac{\|\boldsymbol{a}\|}{m_{\boldsymbol{\phi}}}\left\langle\boldsymbol{\phi}, \boldsymbol{X}_{t}\right\rangle$, where $m_{\boldsymbol{\phi}}:=\min \{\phi(i): i \in E\}$. Therefore $\mathbb{P}_{\boldsymbol{x}^{-}}$a.s.

$$
\begin{aligned}
& \lim _{\sigma \rightarrow 0} \lim _{n \rightarrow \infty} \sup _{t \in[n \sigma,(n+1) \sigma]}\left|\mathrm{e}^{-\lambda_{1} t}\left\langle\boldsymbol{M}((n+1) \sigma-t) \boldsymbol{e}_{k}, \boldsymbol{X}_{t}\right\rangle-\mathrm{e}^{-\lambda_{1} t}\left\langle\boldsymbol{e}_{k}, \boldsymbol{X}_{t}\right\rangle\right| \\
\leq & \lim _{\sigma \rightarrow 0} \lim _{n \rightarrow \infty} \sup _{t \in[n \sigma,(n+1) \sigma]} \mathrm{e}^{-\lambda_{1} t}\left\langle\boldsymbol{\phi}, \boldsymbol{X}_{t}\right\rangle \frac{\left\|\boldsymbol{M}((n+1) \sigma-t) \boldsymbol{e}_{k}-\boldsymbol{e}_{k}\right\|}{m_{\boldsymbol{\phi}}} \\
\leq & \left(\lim _{\sigma \rightarrow 0} \lim _{n \rightarrow \infty} \sup _{t \in[n \sigma,(n+1) \sigma]} W_{t}\right)\left(\lim _{\sigma \rightarrow 0} \sup _{u \in[0, \sigma]} \frac{\left\|\boldsymbol{M}(u) \boldsymbol{e}_{k}-\boldsymbol{e}_{k}\right\|}{m_{\boldsymbol{\phi}}}\right)=0 .
\end{aligned}
$$

So, in order to have our result, it is enough to prove that

$$
\lim _{\sigma \rightarrow 0} \lim _{n \rightarrow \infty} \sup _{t \in[n \sigma,(n+1) \sigma]} \mathrm{e}^{-\lambda_{1} t}\left\langle\boldsymbol{M}((n+1) \sigma-t) \boldsymbol{e}_{k}, \boldsymbol{X}_{t}\right\rangle=\widehat{\phi}(k) W_{\infty} \quad \mathbb{P}_{\boldsymbol{x}} \text { a.s. }
$$


By applying equation (1.7) to $\boldsymbol{e}_{k}$ and $n \sigma \leq t \leq(n+1) \sigma$, we obtain

$$
\begin{aligned}
& \mathrm{e}^{-\lambda_{1} t}\left\langle\boldsymbol{M}((n+1) \sigma-t) \boldsymbol{e}_{k}, \boldsymbol{X}_{t}\right\rangle \\
= & \mathrm{e}^{-\lambda_{1} t}\left\langle\boldsymbol{M}(\sigma) \boldsymbol{e}_{k}, \boldsymbol{X}_{n \sigma}\right\rangle \\
& +\mathrm{e}^{-\lambda_{1} t} \sum_{i \in E} \int_{n \sigma}^{t}\left[\boldsymbol{M}((n+1) \sigma-u) \boldsymbol{e}_{k}\right]_{i} \sqrt{2 c_{i} X_{u, i}} \mathrm{~d} W_{u, i} \\
& +\mathrm{e}^{-\lambda_{1} t} \sum_{i \in E} \int_{n \sigma}^{t} \int_{\mathbb{R}_{+}^{d}} \int_{0}^{\infty}\left\langle\boldsymbol{M}((n+1) \sigma-u) \boldsymbol{e}_{k}, \boldsymbol{z}\right\rangle \mathbf{1}_{\left\{l \leq X_{u-, i}\right\}} \widetilde{N}_{i}^{(1)}(\mathrm{d} u, \mathrm{~d} \boldsymbol{z}, \mathrm{d} l) \\
& +\mathrm{e}^{-\lambda_{1} t} \sum_{i \in E} \int_{n \sigma}^{t} \int_{\mathbb{R}_{+}^{d}} \int_{0}^{\infty}\left\langle\boldsymbol{M}((n+1) \sigma-u) \boldsymbol{e}_{k}, \boldsymbol{z}\right\rangle \mathbf{1}_{\left\{l \leq X_{u-, i}\right\}} \widetilde{N}_{i}^{(2)}(\mathrm{d} u, \mathrm{~d} \boldsymbol{z}, \mathrm{d} l) \\
= & : \mathrm{e}^{-\lambda_{1} t}\left\langle\boldsymbol{M}(\sigma) \boldsymbol{e}_{k}, \boldsymbol{X}_{n \sigma}\right\rangle+C_{n, t}^{\sigma}\left(\boldsymbol{e}_{k}\right)+S_{n, t}^{\sigma}\left(\boldsymbol{e}_{k}\right)+B_{n, t}^{\sigma}\left(\boldsymbol{e}_{k}\right) .
\end{aligned}
$$

By the result for lattice times (Proposition 3.2), $\mathbb{P}_{\boldsymbol{x}}$ a.s.

$$
\begin{aligned}
\lim _{\sigma \rightarrow 0} \lim _{n \rightarrow \infty} \sup _{t \in[n \sigma,(n+1) \sigma]} \mathrm{e}^{-\lambda_{1} t}\left\langle\boldsymbol{M}(\sigma) \boldsymbol{e}_{k}, \boldsymbol{X}_{n \sigma}\right\rangle & =\lim _{\sigma \rightarrow 0} \lim _{n \rightarrow \infty} \mathrm{e}^{-\lambda_{1} \sigma n}\left\langle\boldsymbol{M}(\sigma) \boldsymbol{e}_{k}, \boldsymbol{X}_{n \sigma}\right\rangle \\
& =\widehat{\phi}(k) W_{\infty} .
\end{aligned}
$$

Hence, to complete the proof, we only need to prove that the last three terms on the right-hand side of (3.9) converge uniformly for $t \in[n \sigma,(n+1) \sigma]$ first as $n \rightarrow \infty$ and then $\sigma \rightarrow 0$.

(i) Convergence of $C_{n, t}^{\sigma}\left(\boldsymbol{e}_{k}\right)$ : We show that for any $k \in E$ and $\boldsymbol{x} \in \mathbb{R}_{+}^{d}$,

$$
\lim _{\sigma \rightarrow 0} \lim _{n \rightarrow \infty} \sup _{t \in[n \sigma,(n+1) \sigma]} C_{n, t}^{\sigma}\left(\boldsymbol{e}_{k}\right)=0 \quad \mathbb{P}_{\boldsymbol{x}} \text { a.s. }
$$

Recall the definition of the martingale $C_{t}^{(n, 1, \sigma)}$ given by (3.2). And note that

$$
\left|C_{n, t}^{\sigma}\left(\boldsymbol{e}_{k}\right)\right| \leq \mathrm{e}^{\lambda_{1} \sigma}\left|C_{t}^{(n, 1, \sigma)}\right|
$$

Then, by the maximal inequality for martingales, for all $\epsilon>0$ we have that for

$$
\begin{aligned}
\mathbb{P}_{\boldsymbol{x}}\left(\sup _{t \in[n \sigma,(n+1) \sigma]}\left|C_{n, t}^{\sigma}\left(\boldsymbol{e}_{k}\right)\right|>\epsilon\right) & \leq \mathbb{P}_{\boldsymbol{x}}\left(\sup _{t \in[n \sigma,(n+1) \sigma]} \mathrm{e}^{\lambda_{1} \sigma}\left|C_{t}^{(n, 1, \sigma)}\right|>\epsilon\right) \\
& \leq \frac{\mathrm{e}^{2 \lambda_{1} \sigma}}{\epsilon^{2}} \mathbb{E}_{\boldsymbol{x}}\left[\left|C_{(n+1) \sigma}^{(n, 1, \sigma)}\right|^{2}\right]
\end{aligned}
$$

Note that $C_{(n+1) \sigma}^{(n, 1, \sigma)}=C_{n \sigma,(n+1) \sigma}\left(\boldsymbol{e}_{k}\right)$, therefore by (3.3),

$$
\sum_{n=1}^{\infty} \mathbb{P}_{\boldsymbol{x}}\left(\sup _{t \in[n \sigma,(n+1) \sigma]}\left|C_{n, t}^{\sigma}\left(\boldsymbol{e}_{k}\right)\right|>\epsilon\right)<\infty .
$$

By Borel-Cantelli we have the result.

(ii) Convergence of $S_{n, t}^{\sigma}\left(\boldsymbol{e}_{k}\right)$ : We show that, if ( $x \log x$ condition) holds, then for any $k \in E$ and $\boldsymbol{x} \in \mathbb{R}_{+}^{d}$,

$$
\lim _{\sigma \rightarrow 0} \lim _{n \rightarrow \infty} \sup _{t \in[n \sigma,(n+1) \sigma]} S_{n, t}^{\sigma}\left(\boldsymbol{e}_{k}\right)=0 \quad \mathbb{P}_{\boldsymbol{x}} \text { a.s. }
$$

The proof is analogous to the previous one. But this time we use the martingale $S_{t}^{(n, 1, \sigma)}$ given by (3.4) and equation (3.6). 
(iii) Convergence of $B_{n, t}^{\sigma}\left(\boldsymbol{e}_{k}\right)$ : We show that, if ( $x \log x$ condition) holds, then for any $k \in E$ and $\boldsymbol{x} \in \mathbb{R}_{+}^{d}$,

$$
\lim _{\sigma \rightarrow 0} \lim _{n \rightarrow \infty} \sup _{t \in[n \sigma,(n+1) \sigma]} B_{n, t}^{\sigma}\left(\boldsymbol{e}_{k}\right)=0 \quad \mathbb{P}_{\boldsymbol{x}} \text { a.s. }
$$

By inequality (3.5),

$$
\begin{aligned}
\left|B_{n, t}^{\sigma}\left(\boldsymbol{e}_{k}\right)\right| & \leq C \mathrm{e}^{-\lambda_{1}(t-(n+1) \sigma)} \times \\
& \sum_{i \in E} \int_{n \sigma}^{t} \int_{\mathbb{R}_{+}^{d}} \int_{0}^{\infty} \mathrm{e}^{-\lambda u}\langle\mathbf{1}, \boldsymbol{z}\rangle \mathbf{1}_{\left\{r \leq X_{u, i}\right\}} \mathbf{1}_{\left\{\langle\mathbf{1}, \boldsymbol{z}\rangle>\mathrm{e}^{\lambda_{1} u}\right\}}\left(\mathrm{d} N_{i}+\mathrm{d} r \mu_{i}(\mathrm{~d} \boldsymbol{z}) \mathrm{d} u\right) \\
& \leq C \mathrm{e}^{-\lambda_{1}(t-(n+1) \sigma)} \times \\
& \sum_{i \in E} \int_{n \sigma}^{\infty} \int_{\mathbb{R}_{+}^{d}} \int_{0}^{\infty} \mathrm{e}^{-\lambda u}\langle\mathbf{1}, \boldsymbol{z}\rangle \mathbf{1}_{\left\{r \leq X_{u, i}\right\}} \mathbf{1}_{\left\{\langle\mathbf{1}, \boldsymbol{z}\rangle>\mathrm{e}^{\lambda_{1} u}\right\}}\left(\mathrm{d} N_{i}+\mathrm{d} r \mu_{i}(\mathrm{~d} \boldsymbol{z}) \mathrm{d} u\right) .
\end{aligned}
$$

Then,

$$
\begin{aligned}
& \sup _{t \in[n \sigma,(n+1) \sigma]}\left|B_{n, t}^{\sigma}\left(\boldsymbol{e}_{k}\right)\right| \\
& \leq C \mathrm{e}^{\lambda_{1} \sigma} \sum_{i \in E} \int_{n \sigma}^{\infty} \int_{\mathbb{R}_{+}^{d}} \int_{0}^{\infty} \mathrm{e}^{-\lambda u}\langle\mathbf{1}, \boldsymbol{z}\rangle \mathbf{1}_{\left\{r \leq X_{u, i}\right\}} \mathbf{1}_{\left\{\langle\mathbf{1}, \boldsymbol{z}\rangle>\mathrm{e}^{\lambda_{1} u}\right\}}\left(\mathrm{d} N_{i}+\mathrm{d} r \mu_{i}(\mathrm{~d} \boldsymbol{z}) \mathrm{d} u\right) .
\end{aligned}
$$

The claim is true by following the same steps after equation (3.7). We omit the details here.

Putting the above three conclusions together, we now conclude

$$
\lim _{t \rightarrow \infty} \mathrm{e}^{-\lambda_{1} t}\left\langle\boldsymbol{e}_{k}, \boldsymbol{X}_{t}\right\rangle=\widehat{\phi}(k) W_{\infty}, \quad \mathbb{P}_{\boldsymbol{x}} \text { a.s. }
$$

as required.

\section{Acknowledgements}

A.K. and S.P. would like to acknowledge support from a Royal Society Newton International Fellowship held by S.P. and the Advanced Newton Fellowship held collaboratively between A.K. and J. C. Pardo. Y.-X.R. would like to acknowledge support from NSFC (Grant No. 11671017 and 11731009), and LMEQF.

\section{References}

K. B. Athreya and P. E. Ney. Branching processes. Dover Publications, Inc., Mineola, NY (2004). ISBN 0-486-43474-5. MR2047480.

M. Barczy, Z. Li and G. Pap. Stochastic differential equation with jumps for multitype continuous state and continuous time branching processes with immigration. ALEA Lat. Am. J. Probab. Math. Stat. 12 (1), 129-169 (2015). MR3340375.

M. Barczy and G. Pap. Asymptotic behavior of critical, irreducible multi-type continuous state and continuous time branching processes with immigration. Stoch. Dyn. 16 (4), 1650008, 30 (2016). MR3494683.

Z.-Q. Chen, Y.-X. Ren and R. Song. $L \log L$ criterion for a class of multitype superdiffusions with nonlocal branching mechanism $(2018+)$. To appear in Sci. China Math. 
R. M. Dudley. Real analysis and probability, volume 74 of Cambridge Studies in Advanced Mathematics. Cambridge University Press, Cambridge (2002). ISBN 0-521-00754-2. MR1932358.

E. B. Dynkin. Branching particle systems and superprocesses. Ann. Probab. 19 (3), 1157-1194 (1991). MR1112411.

E. B. Dynkin. Diffusions, superdiffusions and partial differential equations, volume 50 of American Mathematical Society Colloquium Publications. American Mathematical Society, Providence, RI (2002). ISBN 0-8218-3174-7. MR1883198.

E. B. Dynkin and S. E. Kuznetsov. $\mathbb{N}$-measures for branching exit Markov systems and their applications to differential equations. Probab. Theory Related Fields 130 (1), 135-150 (2004). MR2092876.

A. Kyprianou and S. Palau. Extinction properties of multi-type continuous-state branching processes (2018+). To appear in Stoc. Processes and their Applications.

Z. Li. Measure-valued branching Markov processes. Probability and its Applications (New York). Springer, Heidelberg (2011). ISBN 978-3-642-15003-6. MR2760602.

Y.-X. Ren, R. Song and T. Yang. Spine decomposition and $L \log L$ criterion for superprocesses with non-local branching mechanisms. ArXiv Mathematics $e$ prints (2016). arXiv: 1609.02257. 